\title{
OPEN Procedure via cross-Kerr nonlinearities for encoding single logical qubit information onto four-photon decoherence-free states
}

\author{
Jino $\mathrm{Heo}^{1}$ \& Seong-Gon $\mathrm{Choi}^{1,2 \bowtie}$
}

We propose a photonic procedure using cross-Kerr nonlinearities (XKNLs) to encode single logical qubit information onto four-photon decoherence-free states. In quantum information processing, a decoherence-free subspace can secure quantum information against collective decoherence. Therefore, we design a procedure employing nonlinear optical gates, which are composed of XKNLs, quantum bus beams, and photon-number-resolving measurements with linear optical devices, to conserve quantum information by encoding quantum information onto four-photon decoherence-free states (single logical qubit information). Based on our analysis in quantifying the affection (photon loss and dephasing) of the decoherence effect, we demonstrate the experimental condition to acquire the reliable procedure of single logical qubit information having the robustness against the decoherence effect.

The influence of decoherence, nonunitary process, is one of the most significant obstacles hindering the reliable performance of various quantum information processing schemes, such as quantum communication ${ }^{1-8}$, quantum entanglement ${ }^{9-14}$, and quantum computation ${ }^{15-22}$. Therefore, the influence of decoherence should be reduced via active processes (quantum error corrections ${ }^{23-25}$, entanglement purification $\mathrm{s}^{26-28}$, and entanglement concentration $\mathrm{s}^{30,31}$ ) or passive processes (decoherence-free subspaces ${ }^{32-36}$ ).

In particular, utilizing a decoherence-free subspace prevents collective decoherence ${ }^{32-36}$ which occurs the identical decoherence occurring in each qubit in a system to be spread from one subspace to another subspace in a system when uncontrolled interactions between a system and environment affect the schemes of quantum information processing. Applications (passive processes) ${ }^{37-48}$ employing a decoherence-free subspace can provide immunity against collective decoherence ${ }^{32-34}$. For the passive process, a simple method is to encode quantum information onto two-qubit systems as a singlet state ${ }^{36}$ or three-qubit systems as an entangled $W$ state $^{12,14,49,50}$, and a three-qubit decoherence-free state ${ }^{37-41,51}$. However, applications $s^{12,14,36-41,49-51}$ using two- or three-qubit systems can guarantee only a limited effect for maintaining the coherence of quantum information from the influence of collective decoherence in quantum channels. Hence, four-qubit decoherence-free subspaces, passive processes, utilizing various physical resources have been proposed to enhance the efficiency of coherent quantum information, e.g., linear optics with post-selections $s^{41}$, spontaneous parametric down conversions ${ }^{52,53}$, source of entangled state ${ }^{54,55}$, and cavity-QED ${ }^{42,43,48}$.

For the design of quantum information processing schemes, including passive processes, cross-Kerr nonlinearity (XKNL) ${ }^{56-59}$ is an appropriate candidate. Quantum controlled operations using XKNLs have been performed to implement various quantum information processing schemes by the indirect interaction between photons, signal systems, and probe beams, ancillary systems: coherent state, based on quantum non-demolition detections ${ }^{10,12,14,16,18,56-64}$. However, the decoherence effect (photon loss and dephasing) ${ }^{57-59,63,65}$, which results in the evolution from a quantum pure state to a mixed (classical) state, is inevitable when nonlinear optical gates via XKNLs are operated. To utilize quantum bus (qubus) beams and photon-number-resolving (PNR)

${ }^{1}$ Research Institute for Computer and Information Communication (RICIC), Chungbuk National University, Chungdae-ro 1, Seowon-Gu, Cheongju, Republic of Korea. ${ }^{2}$ College of Electrical and Computer Engineering, Chungbuk National University, Chungdae-ro 1, Seowon-Gu, Cheongju, Republic of Korea. ${ }^{\square}$ email: choisg@ cbnu.ac.kr 


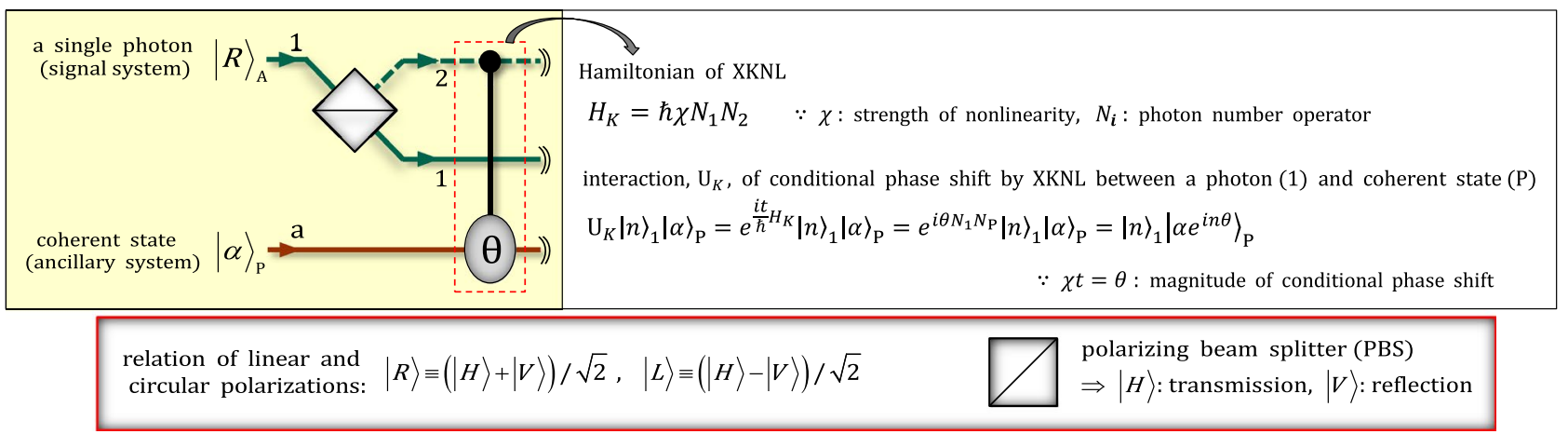

Figure 1. Schematic diagram of the interaction of XKNL: In the Figure, this interaction, conditional phase shift $\theta$ into the phase space of the coherent state $\mathrm{P},|\alpha\rangle_{\mathrm{P}}$, is induced by the polarization $|V\rangle_{\mathrm{P}}$ (path 2) of the photon $\mathrm{A}$ in the Kerr medium. Here, the photon and coherent state $\mathrm{P}$ play the roles of the control qubit, signal system $|V\rangle_{\mathrm{P}}^{2}$, and target qubit, ancillary system $\left|\alpha e^{i \theta}\right\rangle_{\mathrm{P}}^{\mathrm{a}}$, respectively.

measurements ${ }^{10,12,14,16,18,66}$ in nonlinear optical gates via XKNLs with a strong amplitude of coherent states (qubus beams), the decoherence effect should be reduced ${ }^{57-59}$.

In this study, we designed a photonic procedure based on nonlinear optical gates using XKNLs, qubus beams, and PNR measurements to encode quantum information onto four-photon decoherence-free states (single logical qubit information) to achieve robustness against collective decoherence ${ }^{32-34}$. Subsequently, using XKNLs, we quantified the efficiencies and performances of nonlinear optical gates under the decoherence effect (photon loss and dephasing ${ }^{57-59,63,65}$ ). In addition, we derived an experimental condition to reduce the decoherence effect in nonlinear optical gates.

We demonstrate that the proposed procedure for generating single logical qubit information (quantum information on four-photon decoherence-free states) with immunity against collective decoherence can be realized experimentally and that it is robust against the decoherence effect (photon loss and dephasing).

\section{Optical procedure via XKNLs for single logical qubit information}

Four-qubit decoherence-free state. To prevent quantum information in qubits from being affected by collective decoherence ${ }^{32-34}$, logical qubits using decoherence-free subspaces ${ }^{37-48}$ have been utilized. Herein, logical qubits $\left\{\left|0_{L}\right\rangle,\left|1_{L}\right\rangle\right\}$ based on the four-qubit decoherence-free state are expressed as

$$
\begin{aligned}
\left|0_{\mathrm{L}}\right\rangle_{1234} & \equiv \frac{1}{2}(|0101\rangle+|1010\rangle-|0110\rangle-|1001\rangle)_{1234}=\frac{1}{\sqrt{2}}(|01\rangle-|10\rangle)_{12} \otimes \frac{1}{\sqrt{2}}(|01\rangle-|10\rangle)_{34}, \\
\left|1_{\mathrm{L}}\right\rangle_{1234} & \equiv \frac{1}{\sqrt{12}}(2|0011\rangle+2|1100\rangle-|0101\rangle-|1010\rangle-|0110\rangle-|1001\rangle)_{1234} \\
& =\frac{1}{\sqrt{3}}\left[(|0011\rangle+|1100\rangle)_{1234}-\frac{1}{\sqrt{2}}(|01\rangle+|10\rangle)_{12} \otimes \frac{1}{\sqrt{2}}(|01\rangle+|10\rangle)_{34}\right] .
\end{aligned}
$$

Using the logical qubits in Eq. (1) (four-qubit decoherence-free states), we can encode arbitrary quantum information to acquire immunity against collective decoherence, as $\left|\phi_{\mathrm{L}}\right\rangle=\alpha\left|0_{\mathrm{L}}\right\rangle+\beta\left|1_{\mathrm{L}}\right\rangle$ with $|\alpha|^{2}+|\beta|^{2}=1$ (single logical qubit information).

Interaction of XKNL. For the interaction, $\mathrm{U}_{K}$, in Kerr medium, $\mathrm{XKNL}$, which can be employed to realize the diverse quantum information processing schemes, the interaction between a photon A, signal system, and coherent state $\mathrm{P}$, ancillary system, to induce a phase shift $\theta$ by the Kerr medium is described in Fig. 1. For example, we assume an input state, $|R\rangle_{\mathrm{A}} \otimes|\alpha\rangle_{\mathrm{P}}$, to represent the interaction of the XKNL where $|\alpha\rangle=e^{-|\alpha|^{2} / 2} \sum_{n=0}^{\infty} \frac{\alpha^{n}}{\sqrt{n !}}|n\rangle$. After the input state passes through a polarizing beam splitter (PBS), the state is transformed by the interaction (dotted-red box in Fig. 1) of XKNL, as follows:

$$
|R\rangle_{\mathrm{A}}^{1} \otimes|\alpha\rangle_{\mathrm{P}}^{\mathrm{a}} \stackrel{\mathrm{PBS}}{\rightarrow} \frac{1}{\sqrt{2}}\left(|H\rangle_{\mathrm{A}}^{1}+|V\rangle_{\mathrm{A}}^{2}\right) \otimes|\alpha\rangle_{\mathrm{P}}^{\mathrm{a}} \stackrel{\mathrm{U}_{K}}{\rightarrow} \frac{1}{\sqrt{2}}\left(|H\rangle_{\mathrm{A}}^{1} \otimes|\alpha\rangle_{\mathrm{P}}^{\mathrm{a}}+\mathrm{U}_{K}|V\rangle_{\mathrm{A}}^{2} \otimes|\alpha\rangle_{\mathrm{P}}^{\mathrm{a}}\right)=\frac{1}{\sqrt{2}}\left(|H\rangle_{\mathrm{A}}^{1} \otimes|\alpha\rangle_{\mathrm{P}}^{\mathrm{a}}+|V\rangle_{\mathrm{A}}^{2} \otimes\left|\alpha e^{i \theta}\right\rangle_{\mathrm{P}}^{\mathrm{a}}\right),
$$

where the operations of the interaction, conditional phase shift, by XKNL and the PBS are described in Fig. 1. As described in Fig. 1 and Eq. (2), the phase of ancillary system, $|\alpha\rangle_{\mathrm{P}}^{\mathrm{a}}$, is changed, according to the state, $|H\rangle_{\mathrm{A}}^{1}$ or $|V\rangle_{\mathrm{A}}^{2}$, of signal system via the interaction of XKNL. From this interaction, or procedure, we can realize quantum non-demolition detections ${ }^{10,12,14,16,18,56-66}$, which can obtain the information of signal system by the indirect detection in ancillary system, to utilize the various procedure for quantum information processing. For the interaction of XKNLs, the magnitude of XKNL can obtain to $\sim 10^{-2}$, due to the electromagnetically induced transparency ${ }^{67,68}$. Recently, to the measurement-induced quantum operation on weak quantum states of light ${ }^{69}$ can generate a strong XKNL at the single photon level for the applicable quantum information processing. Also, for the Hamiltonian, $H_{K}=\hbar \chi N_{1} N_{2}$, of XKNL with $\chi=\left(g_{1}^{2} g_{2}^{2}\right) /\left(\Delta \Omega_{c}^{2}\right)$, the scheme ${ }^{70}$ have been designed in 


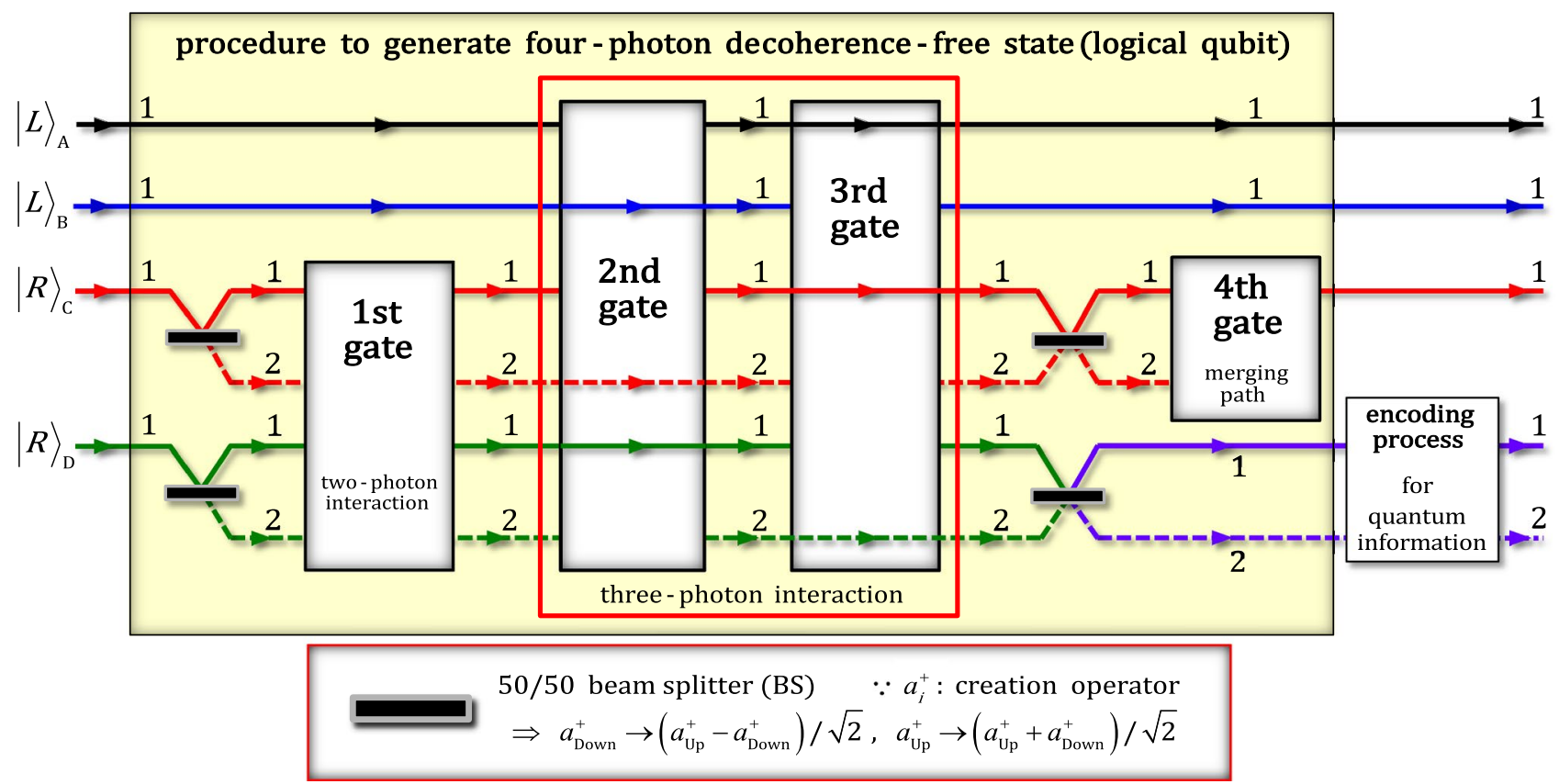

Figure 2. Procedure via XKNLs for single logical qubit information: First part is to generate the superposition of four-photon decoherence-free states. In this part, two-photon interaction of XKNLs is used in the first gate, and the fourth gate (via XKNLs) can merge photon paths. Meanwhile, the second and third gates are operated by three-photon interactions of XKNLs. In the second part, the encoding process can encode (arbitrary) quantum information onto four-photon decoherence-free states, which are output states of the first part.

circuit QED where $g_{i}$ is coupling strength, $\Omega_{c}$ is the transition strength between levels driven by a classical pump field with $\Delta$, detuning. This can show the Kerr medium can be substituted by circuit QED as the proposition ${ }^{71}$ in which the non-maximal entangled states of photons can be concentrated.

Procedure via XKNLs for single logical qubit information. Our procedure pertaining to single logical qubit information comprises two parts: in the first part, four-photon decoherence-free states (the superposition of logical qubits) are generated; in the second part, quantum information is encoded, as described in Fig. 2. In this procedure, all gates, first, second, third, fourth, and final gates, employ the interactions of XKNLs, qubus beams, and PNR measurements. For the single logical qubit information, we prepared the initial state as $\left|\psi_{\text {in }}\right\rangle_{\mathrm{ABCD}}^{1111}=|L\rangle_{\mathrm{A}}^{1} \otimes|L\rangle_{\mathrm{B}}^{1} \otimes|R\rangle_{\mathrm{C}}^{1} \otimes|R\rangle_{\mathrm{D}}^{1}$, where $|R\rangle$-right; $|L\rangle$-left and linear $|H\rangle$-horizontal; $|V\rangle$-vertical represent the circular and linear polarizations of photon, respectively (Fig. 1). After the initial state, $\left|\psi_{\text {in }}\right\rangle_{\mathrm{ABCD}}^{1111}$ passes two 50/50 beam splitters (BSs), and the four-photon state $\left|\psi_{0}\right\rangle_{\mathrm{ABCD}}$ can be expressed as

$$
\left|\psi_{0}\right\rangle_{\mathrm{ABCD}}=|L\rangle_{\mathrm{A}}^{1} \otimes|L\rangle_{\mathrm{B}}^{1} \otimes\left(|R\rangle_{\mathrm{C}}^{1}+|R\rangle_{\mathrm{C}}^{2}\right) / \sqrt{2} \otimes\left(|R\rangle_{\mathrm{D}}^{1}+|R\rangle_{\mathrm{D}}^{2}\right) / \sqrt{2}
$$

where the operation of the BS is illustrated in Fig. 2.

In the first gate, two-photon interactions between photons $\mathrm{C}$ and $\mathrm{D}$, shown in Fig. 3, four conditional phase shifts $\theta$ by XKNLs, two linear phase shifts $-\theta$, qubus beams (two BSs and PNR measurement), and feed-forward (phase shifter and path switch) were exploited for a controlled operation between photons C and D. After the state $\left|\psi_{0}\right\rangle_{\mathrm{ABCD}}$ passes through the first gate, the pre-measurement (before PNR measurement) state $\left|\psi_{0}^{\prime}\right\rangle{ }_{\mathrm{ABCD}}$ can be expressed as

$$
\begin{aligned}
\left|\psi_{0}^{\prime}\right\rangle_{\mathrm{ABCD}}= & |L\rangle_{\mathrm{A}}^{1}|L\rangle_{\mathrm{B}}^{1} \otimes\left[\frac{1}{\sqrt{2}}\left(\frac{1}{\sqrt{2}}|R\rangle_{\mathrm{C}}^{1}|R\rangle_{\mathrm{D}}^{1}+\frac{1}{\sqrt{2}}|R\rangle_{\mathrm{C}}^{2}|R\rangle_{\mathrm{D}}^{2}\right) \otimes|\alpha\rangle_{\mathrm{P}}^{\mathrm{a}}|0\rangle_{\mathrm{P}}^{\mathrm{b}}\right. \\
& \left.+\frac{1}{\sqrt{2}} e^{-\frac{(\alpha \sin \theta)^{2}}{2}} \sum_{n=0}^{\infty} \frac{(i \alpha \sin \theta)^{n}}{\sqrt{n !}}\left(\frac{1}{\sqrt{2}}|R\rangle_{\mathrm{C}}^{1}|R\rangle_{\mathrm{D}}^{2}+\frac{(-1)^{n}}{\sqrt{2}}|R\rangle_{\mathrm{C}}^{2}|R\rangle_{\mathrm{D}}^{1}\right) \otimes|\alpha \cos \theta\rangle_{\mathrm{P}}^{\mathrm{a}}|n\rangle_{\mathrm{P}}^{\mathrm{b}}\right],
\end{aligned}
$$

where $|\alpha\rangle_{\mathrm{P}}$ is the coherent state, probe beam: ancillary system. The operation of the BS in the qubus beam (coherent state) is shown in Fig. 3. $| \pm i \alpha \sin \theta\rangle_{\mathrm{P}}=e^{-(\alpha \sin \theta)^{2} / 2} \sum_{n=0}^{\infty} \frac{( \pm i \alpha \sin \theta)^{n}}{\sqrt{n !}}|n\rangle_{\mathrm{P}}$ for $\alpha \in \mathbb{R}$. Subsequently, by PNR measurement on path $\mathrm{b}$ of the qubus beams, if the outcome is $0\left(|0\rangle_{\mathrm{P}}^{\mathrm{b}}\right.$ : no detection), then the output state, $\left|\psi_{1}\right\rangle_{\mathrm{ABCD}}$ of the first gate can be obtained as $\left|\psi_{1}\right\rangle_{\mathrm{ABCD}}=|L\rangle_{\mathrm{A}}^{1}|L\rangle_{\mathrm{B}}^{1}\left(|R\rangle_{\mathrm{C}}^{1}|R\rangle_{\mathrm{D}}^{1}+|R\rangle_{\mathrm{C}}^{2}|R\rangle_{\mathrm{D}}^{2}\right) / \sqrt{2}$. Meanwhile, if the outcome is $n\left(|n\rangle_{\mathrm{P}}^{\mathrm{b}}: n \neq 0\right)$, then the output state $|L\rangle_{\mathrm{A}}^{1}|L\rangle_{\mathrm{B}}^{1}\left(|R\rangle_{\mathrm{C}}^{1}|R\rangle_{\mathrm{D}}^{2}+(-1)^{n}|R\rangle{ }_{\mathrm{C}}^{2}|R\rangle_{\mathrm{D}}^{1}\right) / \sqrt{2}$ can be transformed to state $\left|\psi_{1}\right\rangle_{\mathrm{ABCD}}$ by feed-forward (phase shifter and path switch), as described in Fig. 3 . 


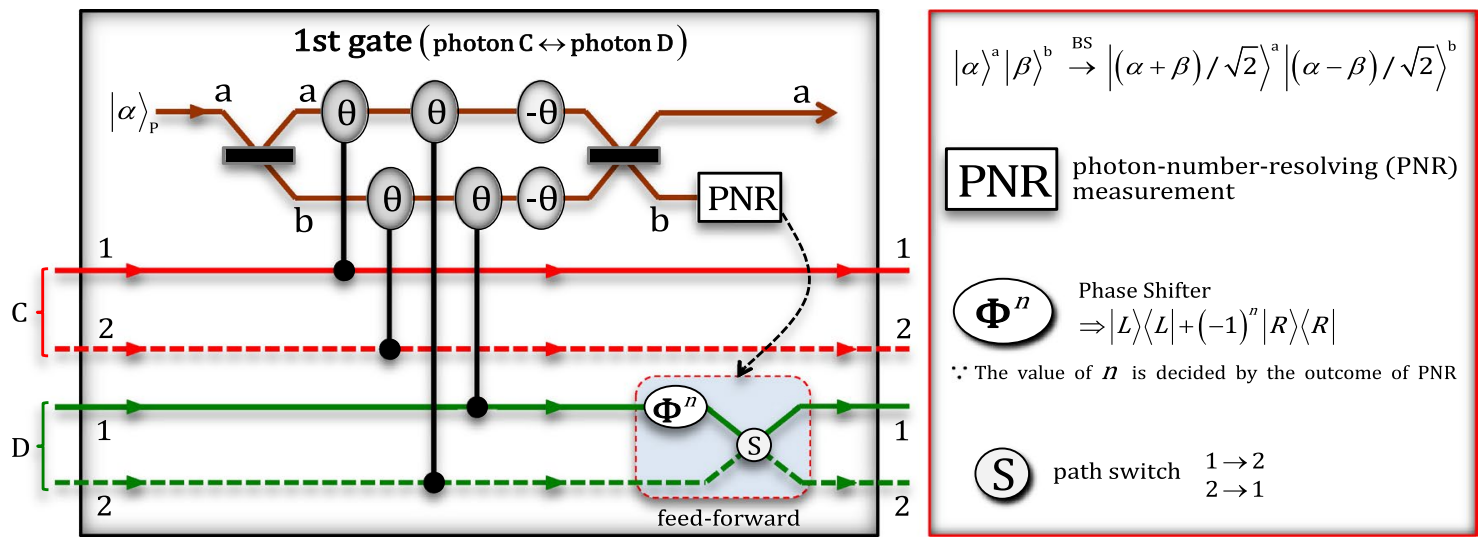

Figure 3. First gate (two-photon interactions between photons $C$ and $D$ ) via XKNLs: For path arrangement of photons $\mathrm{C}$ and $\mathrm{D}$, the first gate comprises XKNLs, qubus beams, PNR measurement, feed-forward, and linear optical devices. After PNR measurement, feed-forward (phase shifter and path switch) on photon D is either operated or not operated, depending on the result (photon number $n$ ) of PNR measurement.

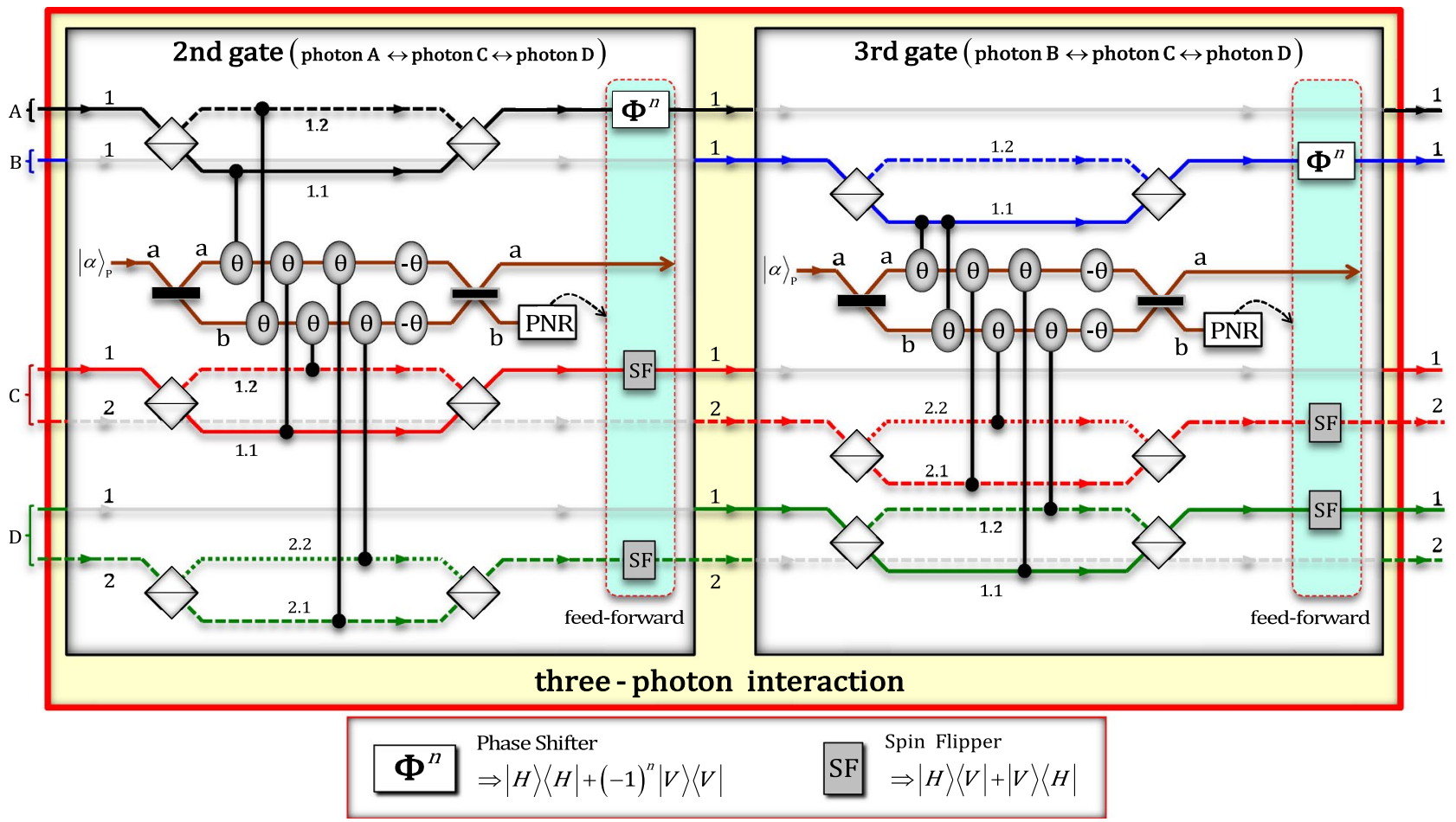

Figure 4. Second and third gates (three-photon interactions) via XKNLs: For controlled operations between three photons, two (second and third) gates consist of XKNLs, qubus beams, PNR measurement, feed-forward, and linear optical devices were used. Feed-forwards (phase shifters and spin flippers) of two gates are either operated or not operated on photons (A, C, and $\mathbf{D}$ : second gate) and (B, C, and $\mathbf{D}$ : third gate) depending on results of PNR measurements.

In the second and third gates, which interact with three-photon, shown in Fig. 4, conditional phase shifts $\theta$ by XKNLs, linear phase shifts $-\theta$, qubus beams (BSs and PNR measurements), feed-forwards (phase shifters and spin flippers), and linear optical devices, including polarizing beam splitters (PBSs), were utilized for controlled operations between three photons, i.e., (A, C, and D: second gate) and (B, C, and D: third gate). After the state $\left|\psi_{1}\right\rangle_{\mathrm{ABCD}}$ (the output state of the first gate) passes through the second gate, the pre-measurement (before PNR measurement) state $\left|\psi_{1}^{\prime}\right\rangle_{\mathrm{ABCD}}$ can be expressed as 


$$
\begin{aligned}
\left|\psi_{1}^{\prime}\right\rangle_{\mathrm{ABCD}}= & \frac{1}{\sqrt{2}}\left\{\frac{1}{2}|H\rangle_{\mathrm{A}}^{1}|L\rangle_{\mathrm{B}}^{1}\left(|V\rangle_{\mathrm{C}}^{1}|R\rangle_{\mathrm{D}}^{1}+|R\rangle_{\mathrm{C}}^{2}|V\rangle_{\mathrm{D}}^{2}\right)-\frac{1}{2}|V\rangle_{\mathrm{A}}^{1}|L\rangle_{\mathrm{B}}^{1}\left(|H\rangle_{\mathrm{C}}^{1}|R\rangle_{\mathrm{D}}^{1}+|R\rangle_{\mathrm{C}}^{2}|H\rangle_{\mathrm{D}}^{2}\right)\right\} \otimes|\alpha\rangle_{\mathrm{P}}^{\mathrm{a}}|0\rangle_{\mathrm{P}}^{\mathrm{b}} \\
& +\frac{1}{\sqrt{2}} e^{-\frac{(\alpha \sin \theta)^{2}}{2}} \sum_{n=0}^{\infty} \frac{(i \alpha \sin \theta)^{n}}{\sqrt{n !}}\left\{\frac{1}{2}|H\rangle_{\mathrm{A}}^{1}|L\rangle_{\mathrm{B}}^{1}\left(|H\rangle_{\mathrm{C}}^{1}|R\rangle_{\mathrm{D}}^{1}+|R\rangle_{\mathrm{C}}^{2}|H\rangle_{\mathrm{D}}^{2}\right)\right. \\
& \left.-\frac{(-1)^{n}}{2}|V\rangle_{\mathrm{A}}^{1}|L\rangle_{\mathrm{B}}^{1}\left(|V\rangle_{\mathrm{C}}^{1}|R\rangle_{\mathrm{D}}^{1}+|R\rangle_{\mathrm{C}}^{2}|V\rangle_{\mathrm{D}}^{2}\right)\right\} \otimes|\alpha \cos \theta\rangle_{\mathrm{P}}^{\mathrm{a}}|n\rangle_{\mathrm{P}}^{\mathrm{b}} .
\end{aligned}
$$

Depending on the PNR measurement result on path $b$ of the qubus beams, if the outcome is $0\left(|0\rangle{ }_{\mathrm{P}}^{\mathrm{b}}\right.$ : no detection), then the output state, $\left|\psi_{2}\right\rangle_{\mathrm{ABCD}}$ of the second gate can be obtained as $\left|\psi_{2}\right\rangle_{\mathrm{ABCD}}=\left\{|H\rangle_{\mathrm{A}}^{1}|L\rangle_{\mathrm{B}}^{1}\left(|V\rangle_{\mathrm{C}}^{1}|R\rangle_{\mathrm{D}}^{1}+|R\rangle_{\mathrm{C}}^{2}|V\rangle_{\mathrm{D}}^{2}\right)-|V\rangle_{\mathrm{A}}^{1}|L\rangle_{\mathrm{B}}^{1}\left(|H\rangle_{\mathrm{C}}^{1}|R\rangle_{\mathrm{D}}^{1}+|R\rangle_{\mathrm{C}}^{2}|H\rangle_{\mathrm{D}}^{2}\right)\right\} / 2$. Otherwise, if $n$ $\left(|n\rangle_{\mathrm{P}}^{\mathrm{b}}: n \neq 0\right)$, then the output state can be changed to $\left|\psi_{2}\right\rangle_{\mathrm{ABCD}}$ by feed-forwards (phase shifter and spin flippers). Subsequently, the state $\left|\psi_{2}\right\rangle_{\mathrm{ABCD}}$ enters the third gate for another controlled operation. After the third gate, the state $\left|\psi_{2}^{\prime}\right\rangle_{\mathrm{ABCD}}$ (before PNR measurement) can be written as

$$
\begin{aligned}
& \left|\psi_{2}^{\prime}\right\rangle{ }_{\mathrm{ABCD}}=\frac{1}{\sqrt{2}}\left[\frac{1}{2 \sqrt{2}}\left\{|H\rangle_{\mathrm{A}}^{1}|H\rangle_{\mathrm{B}}^{1}\left(|V\rangle_{\mathrm{C}}^{1}|V\rangle_{\mathrm{D}}^{1}+|V\rangle_{\mathrm{C}}^{2}|V\rangle_{\mathrm{D}}^{2}\right)-|V\rangle_{\mathrm{A}}^{1}|H\rangle_{\mathrm{B}}^{1}\left(|H\rangle_{\mathrm{C}}^{1}|V\rangle_{\mathrm{D}}^{1}+|V\rangle_{\mathrm{C}}^{2}|H\rangle_{\mathrm{D}}^{2}\right)\right\}\right. \\
& \left.+\frac{1}{2 \sqrt{2}}\left\{|V\rangle_{\mathrm{A}}^{1}|V\rangle_{\mathrm{B}}^{1}\left(|H\rangle_{\mathrm{C}}^{1}|H\rangle_{\mathrm{D}}^{1}+|H\rangle_{\mathrm{C}}^{2}|H\rangle_{\mathrm{D}}^{2}\right)-|H\rangle_{\mathrm{A}}^{1}|V\rangle_{\mathrm{B}}^{1}\left(|H\rangle_{\mathrm{C}}^{2}|V\rangle_{\mathrm{D}}^{2}+|V\rangle_{\mathrm{C}}^{1}|H\rangle{ }_{\mathrm{D}}^{1}\right)\right\}\right] \otimes|\alpha\rangle_{\mathrm{P}}^{\mathrm{a}}|0\rangle_{\mathrm{P}}^{\mathrm{b}} \\
& +\frac{1}{\sqrt{2}} e^{-\frac{(\alpha \sin \theta)^{2}}{2}} \sum_{n=0}^{\infty} \frac{(i \alpha \sin \theta)^{n}}{\sqrt{n !}}\left[\frac{1}{2 \sqrt{2}}\left\{|H\rangle_{\mathrm{A}}^{1}|H\rangle_{\mathrm{B}}^{1}\left(|V\rangle_{\mathrm{C}}^{1}|H\rangle_{\mathrm{D}}^{1}+|H\rangle_{\mathrm{C}}^{2}|V\rangle_{\mathrm{D}}^{2}\right)-|V\rangle_{\mathrm{A}}^{1}|H\rangle_{\mathrm{B}}^{1}\left(|H\rangle_{\mathrm{C}}^{1}|H\rangle_{\mathrm{D}}^{1}+|H\rangle_{\mathrm{C}}^{2}|H\rangle_{\mathrm{D}}^{2}\right)\right\}\right. \\
& \left.+\frac{(-1)^{n}}{2 \sqrt{2}}\left\{|V\rangle_{\mathrm{A}}^{1}|V\rangle_{\mathrm{B}}^{1}\left(|H\rangle_{\mathrm{C}}^{1}|V\rangle_{\mathrm{D}}^{1}+|V\rangle_{\mathrm{C}}^{2}|H\rangle_{\mathrm{D}}^{2}\right)-|H\rangle_{\mathrm{A}}^{1}|V\rangle_{\mathrm{B}}^{1}\left(|V\rangle_{\mathrm{C}}^{1}|V\rangle_{\mathrm{D}}^{1}+|V\rangle_{\mathrm{C}}^{2}|V\rangle_{\mathrm{D}}^{2}\right)\right\}\right] \otimes|\alpha \cos \theta\rangle_{\mathrm{P}}^{\mathrm{a}}|n\rangle_{\mathrm{P}}^{\mathrm{b}} .
\end{aligned}
$$

After the operations applying feed-forwards or not in Fig. 4, owing to the outcome of the PNR measurement, the output state $\left|\psi_{3}\right\rangle_{\mathrm{ABCD}}$ of the third gate can be expressed as

$$
\begin{aligned}
\left|\psi_{3}\right\rangle_{\mathrm{ABCD}}= & \frac{1}{2}\left[|H\rangle_{\mathrm{A}}^{1}|H\rangle_{\mathrm{B}}^{1} \otimes\left(|V\rangle_{\mathrm{C}}^{1}|V\rangle_{\mathrm{D}}^{1}+|V\rangle_{\mathrm{C}}^{2}|V\rangle_{\mathrm{D}}^{2}\right) / \sqrt{2}-|V\rangle_{\mathrm{A}}^{1}|H\rangle_{\mathrm{B}}^{1} \otimes\left(|H\rangle_{\mathrm{C}}^{1}|V\rangle_{\mathrm{D}}^{1}+|V\rangle_{\mathrm{C}}^{2}|H\rangle_{\mathrm{D}}^{2}\right) / \sqrt{2}\right. \\
& \left.+|V\rangle_{\mathrm{A}}^{1}|V\rangle_{\mathrm{B}}^{1} \otimes\left(|H\rangle_{\mathrm{C}}^{1}|H\rangle_{\mathrm{D}}^{1}+|H\rangle_{\mathrm{C}}^{2}|H\rangle_{\mathrm{D}}^{2}\right) / \sqrt{2}-|H\rangle_{\mathrm{A}}^{1}|V\rangle_{\mathrm{B}}^{1} \otimes\left(|H\rangle_{\mathrm{C}}^{2}|V\rangle_{\mathrm{D}}^{2}+|V\rangle_{\mathrm{C}}^{1}|H\rangle_{\mathrm{D}}^{1}\right) / \sqrt{2}\right] .
\end{aligned}
$$

Subsequently, as described in Fig. 2, two BSs were applied to photons C and D of the output state $\left|\psi_{3}\right\rangle_{\mathrm{ABCD}}$ in Eq. 7. Next, the output state $\left|\psi_{3}\right\rangle_{\mathrm{ABCD}}$ was transformed to state $\left|\psi_{3}^{\prime}\right\rangle_{\mathrm{ABCD}}$ of the superposed (four-photon) decoherence-free states, as follows:

$$
\begin{aligned}
\left|\psi_{3}^{\prime}\right\rangle= & \frac{1}{4 \sqrt{2}}\left[\left\{|H\rangle_{\mathrm{A} C D}^{1}|V\rangle_{\mathrm{B}}^{1}|H\rangle_{\mathrm{C}}^{1}|V\rangle_{\mathrm{D}}^{2}+|V\rangle_{\mathrm{A}}^{1}|H\rangle_{\mathrm{B}}^{1}|V\rangle_{\mathrm{C}}^{1}|H\rangle_{\mathrm{D}}^{2}-|H\rangle_{\mathrm{A}}^{1}|V\rangle_{\mathrm{B}}^{1}|V\rangle_{\mathrm{C}}^{1}|H\rangle_{\mathrm{D}}^{2}-|V\rangle_{\mathrm{A}}^{1}|H\rangle_{\mathrm{B}}^{1}|H\rangle_{\mathrm{C}}^{1}|V\rangle_{\mathrm{D}}^{2}\right\}\right. \\
& +\left\{|H\rangle_{\mathrm{A}}^{1}|V\rangle_{\mathrm{B}}^{1}|H\rangle_{\mathrm{C}}^{2}|V\rangle_{\mathrm{D}}^{1}+|V\rangle_{\mathrm{A}}^{1}|H\rangle_{\mathrm{B}}^{1}|V\rangle_{\mathrm{C}}^{2}|H\rangle_{\mathrm{D}}^{1}-|H\rangle_{\mathrm{A}}^{1}|V\rangle_{\mathrm{B}}^{1}|V\rangle_{\mathrm{C}}^{2}|H\rangle_{\mathrm{D}}^{1}-|V\rangle_{\mathrm{A}}^{1}|H\rangle_{\mathrm{B}}^{1}|H\rangle_{\mathrm{C}}^{2}|V\rangle_{\mathrm{D}}^{1}\right\} \\
& +\left\{2|H\rangle_{\mathrm{A}}^{1}|H\rangle_{\mathrm{B}}^{1}|V\rangle_{\mathrm{C}}^{1}|V\rangle_{\mathrm{D}}^{1}+2|V\rangle_{\mathrm{A}}^{1}|V\rangle_{\mathrm{B}}^{1}|H\rangle_{\mathrm{C}}^{1}|H\rangle_{\mathrm{D}}^{1}-|V\rangle_{\mathrm{A}}^{1}|H\rangle_{\mathrm{B}}^{1}|H\rangle_{\mathrm{C}}^{1}|V\rangle_{\mathrm{D}}^{1}-|H\rangle_{\mathrm{A}}^{1}|V\rangle_{\mathrm{B}}^{1}|V\rangle_{\mathrm{C}}^{1}|H\rangle_{\mathrm{D}}^{1}\right. \\
& \left.-|H\rangle_{\mathrm{A}}^{1}|V\rangle_{\mathrm{B}}^{1}|H\rangle_{\mathrm{C}}^{1}|V\rangle_{\mathrm{D}}^{1}-|V\rangle_{\mathrm{A}}^{1}|H\rangle_{\mathrm{B}}^{1}|V\rangle_{\mathrm{C}}^{1}|H\rangle_{\mathrm{D}}^{1}\right\}+\left\{2|H\rangle_{\mathrm{A}}^{1}|H\rangle_{\mathrm{B}}^{1}|V\rangle_{\mathrm{C}}^{2}|V\rangle_{\mathrm{D}}^{2}+2|V\rangle_{\mathrm{A}}^{1}|V\rangle_{\mathrm{B}}^{1}|H\rangle_{\mathrm{C}}^{2}|H\rangle_{\mathrm{D}}^{2}\right. \\
& \left.\left.\left.-|V\rangle_{\mathrm{A}}^{1}|H\rangle_{\mathrm{B}}^{1}|H\rangle_{\mathrm{C}}^{2}|V\rangle_{\mathrm{D}}^{2}-|H\rangle_{\mathrm{A}}^{1}|V\rangle_{\mathrm{B}}^{1}|V\rangle_{\mathrm{C}}^{2}|H\rangle_{\mathrm{D}}^{2}-|H\rangle_{\mathrm{A}}^{1}|V\rangle_{\mathrm{B}}^{1}|H\rangle_{\mathrm{C}}^{2}|V\rangle_{\mathrm{D}}^{2}-|V\rangle_{\mathrm{A}}^{1}|H\rangle_{\mathrm{B}}^{1}|V\rangle_{\mathrm{C}}^{2}|H\rangle_{\mathrm{D}}^{2}\right)\right\}\right] \\
\equiv & \frac{1}{\sqrt{2}}\left(\frac{1}{2}\left|0_{\mathrm{L}}\right\rangle_{\mathrm{ABCD}}^{1112}+\frac{\sqrt{3}}{2}\left|1_{\mathrm{L}}\right\rangle_{\mathrm{ABCD}}^{1111}\right)+\frac{1}{\sqrt{2}}\left(\frac{1}{2}\left|0_{\mathrm{L}}\right\rangle_{\mathrm{ABCD}}^{1121}+\frac{\sqrt{3}}{2}\left|1_{\mathrm{L}}\right\rangle_{\mathrm{ABCD}}^{1122}\right),
\end{aligned}
$$

where we define the polarizations $(|H\rangle$ and $|V\rangle)$ of photons corresponding to states $(|0\rangle$ and $|1\rangle)$ of the qubit as $\{|H\rangle,|V\rangle\} \equiv\{|0\rangle,|1\rangle\}$. Hence, state $\left|\psi_{3}^{\prime}\right\rangle_{\mathrm{ABCD}}$ is the photonic superposition of logical qubits (four-qubit decoherence-free states in Eq. 1), according to the paths of photons $\mathrm{C}$ and $\mathrm{D}$.

In the fourth gate (photon C) shown in Fig. 5, two conditional phase shifts $\theta$ by XKNLs, one linear phase shift $-\theta$, qubus beams (two BSs and PNR measurement), and feed-forward (path switch) were utilized to merge the path of photon $\mathrm{C}$ (path 1 and path $2 \rightarrow$ path 1 ). After state $\left|\psi_{3}^{\prime}\right\rangle_{\mathrm{ABCD}}$ passes through the fourth gate, the premeasurement (before PNR) state $\left|\psi_{3}^{\prime \prime}\right\rangle_{\mathrm{ABCD}}$ can be expressed as

$$
\begin{aligned}
\left|\psi_{3}^{\prime \prime}\right\rangle_{\mathrm{ABCD}}= & \frac{1}{\sqrt{2}}\left(\frac{1}{2}\left|0_{\mathrm{L}}\right\rangle_{\mathrm{ABCD}}^{1112}+\frac{\sqrt{3}}{2}\left|1_{\mathrm{L}}\right\rangle_{\mathrm{ABCD}}^{1111}\right) \otimes|\alpha\rangle_{\mathrm{P}}^{\mathrm{a}}|0\rangle_{\mathrm{P}}^{\mathrm{b}} \\
& +\frac{1}{\sqrt{2}} e^{-\frac{(\alpha \sin \theta)^{2}}{2}} \sum_{n=0}^{\infty} \frac{(-i \alpha \sin \theta)^{n}}{\sqrt{n !}}\left(\frac{1}{2}\left|0_{\mathrm{L}}\right\rangle_{\mathrm{ABCD}}^{1121}+\frac{\sqrt{3}}{2}\left|1_{\mathrm{L}}\right\rangle_{\mathrm{ABCD}}^{1122}\right) \otimes|\alpha \cos \theta\rangle_{\mathrm{P}}^{\mathrm{a}}|n\rangle_{\mathrm{P}}^{\mathrm{b}} .
\end{aligned}
$$

Subsequently, by PNR measurement on path $\mathrm{b}$ of the qubus beams, if the outcome is $0\left(|0\rangle_{\mathrm{P}}^{\mathrm{b}}\right.$ : no detection), then output state is $\left|\psi_{4}\right\rangle_{\mathrm{ABCD}}=\left(\left|0_{\mathrm{L}}\right\rangle_{\mathrm{ABCD}}^{1112}+\sqrt{3}\left|1_{\mathrm{L}}\right\rangle_{\mathrm{ABCD}}^{1111}\right) / 2$. Meanwhile, if the outcome is $n\left(|n\rangle_{\mathrm{P}}^{\mathrm{b}}: n \neq 0\right)$, 


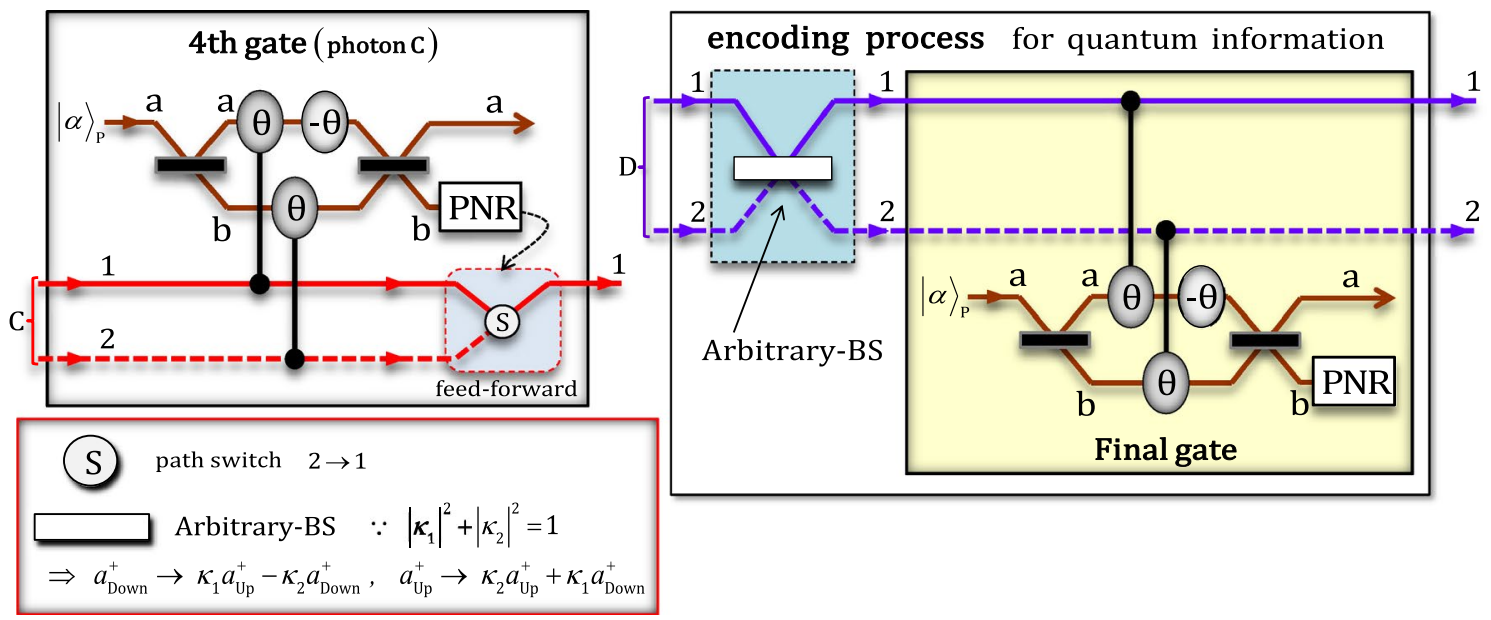

Figure 5. Fourth gate via XKNLs; encoding process with arbitrary-BS and final gate (XKNLs): The fourth gate merged the path of photon C using XKNLs, qubus beams, PNR measurement, and feed-forward (path switch). During encoding, the arbitrary-BS (linear optical device) and final gate (via XKNLs) encode arbitrary quantum information onto four-photon decoherence-free states (single logical qubit information).

then the output state $\left(\left|0_{\mathrm{L}}\right\rangle_{\mathrm{ABCD}}^{1121}+\sqrt{3}\left|1_{\mathrm{L}}\right\rangle_{\mathrm{ABCD}}^{1122}\right) / 2$ can be transformed to state $\left|\psi_{4}\right\rangle_{\mathrm{ABCD}}$ by feed-forward (path switch), as shown in Fig. 5.

The encoding process shown in Fig. 5 comprises two parts (linear: arbitrary-BS, and nonlinear: final gate via XKNLs). To encode arbitrary quantum information for our purposes, communication, computation, teleportation, etc., onto the output state $\left|\psi_{4}\right\rangle_{\mathrm{ABCD}}$ of the fourth gate, we can control the transmission rate $\left(\kappa_{1}\right)$ and reflection rate $\left(\kappa_{2}\right)$ of an arbitrary-BS, in which the operations are expressed as described in Fig. 5. Therefore, after applying the arbitrary-BS to state $\left|\psi_{4}\right\rangle_{\mathrm{ABCD}}=\left(\left|0_{\mathrm{L}}\right\rangle_{\mathrm{ABCD}}^{1112}+\sqrt{3}\left|1_{\mathrm{L}}\right\rangle_{\mathrm{ABCD}}^{1111}\right) / 2$, the encoded (superposition of) state $\left|\psi_{5}\right\rangle_{\mathrm{ABCD}}$ is expressed as

$$
\begin{aligned}
\left|\psi_{5}\right\rangle_{\mathrm{ABCD}} & =\frac{1}{\sqrt{2}}\left[\frac{1}{\sqrt{3\left|\kappa_{2}\right|^{2}+\left|\kappa_{1}\right|^{2}}}\left(\kappa_{1}\left|0_{\mathrm{L}}\right\rangle_{\mathrm{ABCD}}^{1111}+\sqrt{3} \kappa_{2}\left|1_{\mathrm{L}}\right\rangle_{\mathrm{ABCD}}^{1111}\right)-\frac{1}{\sqrt{3\left|\kappa_{1}\right|^{2}+\left|\kappa_{2}\right|^{2}}}\left(\kappa_{2}\left|0_{\mathrm{L}}\right\rangle_{\mathrm{ABCD}}^{1112}-\sqrt{3} \kappa_{1}\left|1_{\mathrm{L}}\right\rangle_{\mathrm{ABCD}}^{1112}\right)\right] \\
& \equiv \frac{1}{\sqrt{2}}\left[\left(\alpha_{1}\left|0_{\mathrm{L}}\right\rangle_{\mathrm{ABCD}}^{1111}+\beta_{1}\left|1_{\mathrm{L}}\right\rangle_{\mathrm{ABCD}}^{1111}\right)-\left(\alpha_{2}\left|0_{\mathrm{L}}\right\rangle_{\mathrm{ABCD}}^{1112}-\beta_{2}\left|1_{\mathrm{L}}\right\rangle_{\mathrm{ABCD}}^{1112}\right)\right],
\end{aligned}
$$

where $\left|\alpha_{i}\right|^{2}+\left|\beta_{i}\right|^{2}=1 . \alpha_{i}$ and $\beta_{i}$ denote the arbitrary information encoded by the arbitrary-BS. Subsequently, through the final gate, the pre-measurement (before PNR measurement) state $\left|\psi_{5}^{\prime}\right\rangle{ }_{\mathrm{ABCD}}$ is expressed as

$$
\begin{aligned}
\left|\psi_{5}^{\prime}\right\rangle_{\mathrm{ABCD}}= & \frac{1}{\sqrt{2}}\left(\alpha_{1}\left|0_{\mathrm{L}}\right\rangle_{\mathrm{ABCD}}^{1111}+\beta_{1}\left|1_{\mathrm{L}}\right\rangle_{\mathrm{ABCD}}^{1111}\right) \otimes|\alpha\rangle_{\mathrm{P}}^{\mathrm{a}}|0\rangle_{\mathrm{P}}^{\mathrm{b}} \\
& -\frac{1}{\sqrt{2}} e^{-\frac{(\alpha \sin \theta)^{2}}{2}} \sum_{n=0}^{\infty} \frac{(-i \alpha \sin \theta)^{n}}{\sqrt{n !}}\left(\alpha_{2}\left|0_{\mathrm{L}}\right\rangle_{\mathrm{ABCD}}^{1112}-\beta_{2}\left|1_{\mathrm{L}}\right\rangle_{\mathrm{ABCD}}^{1112}\right) \otimes|\alpha \cos \theta\rangle_{\mathrm{P}}^{\mathrm{a}}|n\rangle_{\mathrm{P}}^{\mathrm{b}} .
\end{aligned}
$$

After PNR measurement on path b, we can obtain the final state (single logical qubit information), which is the encoded arbitrary information, for the outcomes $(n=0$ or $n \neq 0)$ of the PNR measurement, as follows:

$$
\begin{aligned}
& (n=0) \rightarrow\left|\psi_{\mathrm{f} \_0}\right\rangle_{\mathrm{ABCD}}=\alpha_{1}\left|0_{\mathrm{L}}\right\rangle_{\mathrm{ABCD}}^{1111}+\beta_{1}\left|1_{\mathrm{L}}\right\rangle_{\mathrm{ABCD}}^{1111}, \\
& (n \neq 0) \rightarrow\left|\psi_{\mathrm{f} \_\mathrm{n}}\right\rangle_{\mathrm{ABCD}}=\alpha_{2}\left|0_{\mathrm{L}}\right\rangle_{\mathrm{ABCD}}^{1112}-\beta_{2}\left|1_{\mathrm{L}}\right\rangle_{\mathrm{ABCD}}^{1112},
\end{aligned}
$$

where the final state $\left|\psi_{\mathrm{f}_{-} \mathrm{n}}\right\rangle_{\mathrm{ABCD}}(n \neq 0)$ can be converted to state $\left|\psi_{\mathrm{f}_{-} 0}\right\rangle_{\mathrm{ABCD}}(n=0)$ by applying unitary operations since the transmission rate, $\kappa_{1}$, and reflection rate, $\kappa_{2}$, of an arbitrary-BS are known. Consequently, for the single logical qubit information, the proposed procedure shown in Fig. 2 can encode the arbitrary quantum information $\left(\alpha_{i}\right.$ and $\left.\beta_{i}\right)$ onto four-photon decoherence-free states which are superposed state of $\left|0_{\mathrm{L}}\right\rangle$ and $\left|1_{\mathrm{L}}\right\rangle$, as shown in Eq. (12).

Herein, we propose a procedure comprising nonlinear optical gates, first, second, third, fourth, and final gates via XKNLs, and linear optical devices, including the arbitrary-BS, to encode single logical qubit information onto logical qubits (four-photon decoherence-free states) to protect quantum information against collective decoherence ${ }^{32-34}$. However, the nonlinear optical gates, which are components critical to this procedure (Fig. 2), cannot avoid the influences of photon loss and dephasing induced by the decoherence effect ${ }^{57-59,63,65,72,73}$. Therefore, we should derive the experimental condition to reduce the decoherence effect ${ }^{57-59,63,65}$ based on the master equation ${ }^{74}$ to quantify the efficiency and performance of the nonlinear optical gates. 


\section{Quantification of efficiency and performance of nonlinear optical gates via XKNLs under decoherence effect}

In the proposed procedure, the nonlinear optical gates, first, second, third, fourth, and final gates, using XKNLs are the most important components for generating decoherence-free states (logical qubits) and encoding arbitrary quantum information. Hence, these gates must be highly efficient and reliable when the proposed procedure is implemented in optical fibers ${ }^{72,73}$. Regarding the interaction of XKNLs of these gates, we should derive an experimental condition to decrease the decoherence effect ${ }^{57-59,63,65}$ using the master equation ${ }^{74}$, which can indicate the open quantum system, as follows:

$$
\frac{\partial \rho(t)}{\partial t}=\frac{-i}{\hbar}\left[H_{K}, \rho\right]+\gamma\left[a \rho a^{+}-\frac{1}{2}\left\{a^{+} a \rho+\rho a^{+} a\right\}\right],
$$

where $H_{K}=\hbar \chi N_{1} N_{2}$. The Lindblad operators are $\widehat{J} \rho=\gamma a \rho a^{+}$and $\widehat{L} \rho=-\frac{\gamma}{2}\left(a^{+} a \rho+\rho a^{+} a\right)$, where $\gamma$ is the energy decay rate. The solution to this equation is $\rho(t)=\exp [(\widehat{\mathrm{J}}+\widehat{\mathrm{L}}) \mathrm{t}] \rho(0)$ for time $t(=\theta / \chi)$. Using this solution, we can exploit the process model, which can be used to formulate the influences of photon loss and dephasing of coherent parameters caused by the interaction (conditional phase shift) of XKNLs in nonlinear optical gates under the decoherence effect, as follows:

$$
\left(\hat{D}_{\Delta t} \hat{X}_{\Delta t}\right)^{N}|1\rangle\langle 0|\otimes| \alpha\rangle\left\langle\alpha\left|=\exp \left[-\alpha^{2}\left(1-\mathrm{e}^{-\gamma \Delta \mathrm{t}}\right) \sum_{\mathrm{n}=1}^{\mathrm{N}}\left(1-\mathrm{e}^{\mathrm{in} \Delta \theta}\right) \mathrm{e}^{-\gamma \Delta \mathrm{t}(\mathrm{n}-1)}\right]\right| 1\right\rangle\left\langle 0|\otimes| \Lambda_{\mathrm{t}} \alpha \mathrm{e}^{\mathrm{i} \theta}\right\rangle\left\langle\Lambda_{\mathrm{t}} \alpha\right|,
$$

where $\hat{D}_{t} \hat{X}_{t}=\left(\hat{D}_{\Delta t} \hat{X}_{\Delta t}\right)^{N}$ for $\theta=\chi t=\chi N \Delta t=N \Delta \theta$ owing to an arbitrarily small time $\Delta t(=t / N)$ for obtaining a good approximation ${ }^{57-59,63,65}$. Here, the decoherence $\hat{D}_{t}$ (photon loss and dephasing) and the rate, $\Lambda_{t}=e^{-\gamma t / 2}$ of the remaining photons in the probe beam can be calculated from the solution of the master equation shown in Eq. (13). Furthermore, the operation of the operator $\hat{X}_{t}$ (of the interaction of XKNLs) is expressed as $\left(\hat{X}_{\Delta t}\right)^{N}|1\rangle\langle 0|\otimes| \alpha\rangle\langle\alpha|=| 1\rangle\left\langle 0|\otimes| \alpha e^{i N \Delta \theta}\right\rangle\langle\alpha|=| 1\rangle\left\langle 0|\otimes| \alpha e^{i \theta}\right\rangle\langle\alpha|$ for $|1\rangle$ (one photon) and $|0\rangle$ (zero photon). Using this process model (decoherence: $\hat{D}_{t}$ and interaction of XKNL: $\hat{X}_{t}$ ) of Eqs. (13) and (14), we can quantify the influences of photon loss $\left(\Lambda_{t}=e^{-\gamma t / 2}\right)$ and dephasing of the value of the coherent parameter, i.e., the coefficient of the right-hand side of Eq. (14), induced by the decoherence effect. For the large phase shift $(\approx \pi)$ at room temperature, researchers in Ref. ${ }^{75}$ demonstrated that the implementation of large phase shifts on a single-photon level probe pulse $(1.5 \mu \mathrm{s})$ is mediated by $\mathrm{Rb}^{87}$ vapor in a double- $\Lambda$ atomic configuration to apply to quantum non-demolition detections $10,12,14,16,18,56-64$. Also, for the practical realization of nonlinear optical gates, we should consider the experimental parameters and the features in the optical fibers ${ }^{72,73}$. In commercial fibers, which are pure silica-core fibers with a signal loss of $0.15 \mathrm{~dB} / \mathrm{km}(\chi / \gamma=0.0303)^{73}$, a length of approximately $3000 \mathrm{~km}$ is required to acquire the magnitude of the conditional phase shift, $\theta=\pi$ from XKNLs. Hence, using the process model (Eqs. 13 and 14) with the experimental parameters and features of the optical fiber (length of $3000 \mathrm{~km}$ for $\theta=\pi$ and signal loss of $0.15 \mathrm{~dB} / \mathrm{km}$ ), we can analyze and quantify the efficiencies and performances of the nonlinear optical gates in the proposed procedure for encoding single logical qubit information (Fig. 2).

For the quantification of efficiency in ideal cases without the decoherence effect, we can obtain the error probabilities of nonlinear optical gates from the probabilities of measuring state $|0\rangle_{\mathrm{P}}^{\mathrm{b}}$ (zero photon) in state $| \pm i \alpha \sin \theta\rangle_{\mathrm{P}}^{\mathrm{b}}$ (Eqs. 4, 5, 6, 9, and 11), as follows: $\mathrm{P}_{\mathrm{err}}=\left[\exp \left(-\alpha^{2} \sin ^{2} \theta\right)\right] / 2 \approx\left[\exp \left(-\alpha^{2} \theta^{2}\right)\right] / 2$ for $\alpha^{2} \sin ^{2} \theta \approx \alpha^{2} \theta^{2}$ with a strong amplitude of coherent state and small phase shift magnitude by the XKNL ( $\alpha \gg 1$ and $\theta \ll 1)$. If we do not consider the decoherence effect (ideal case), then the error probabilities of all nonlinear optical gates (first, second, third, fourth, and final) will be identical, as $\mathrm{P}_{\text {err }} \approx\left[\exp \left(-\alpha^{2} \theta^{2}\right)\right] / 2$. In addition, when the parameter is fixed as $\alpha \theta=2.5$, we can acquire highly efficient nonlinear optical gates (first, second, third, fourth, and final) because $\mathrm{P}_{\text {err }}<10^{-3}$.

However, in the practical cases where the decoherence effect is considered, we should recalculate the error probabilities $\left(\mathrm{P}_{\mathrm{err}}^{1 \mathrm{st}}, \mathrm{P}_{\mathrm{err}}^{2 \text { nd }}, \mathrm{P}_{\mathrm{err}}^{3 \mathrm{rd}}, \mathrm{P}_{\mathrm{err}}^{4 \text { th }}\right.$, and $\left.\mathrm{P}_{\mathrm{err}}^{\mathrm{fin}}\right)$ including the photon loss (the rate $\Lambda_{t}=e^{-\gamma t / 2}$ of remaining photons) due to the decoherence effect, as follows:

$$
\begin{aligned}
& \mathrm{P}_{\mathrm{err}}^{1 \mathrm{st}}=\left[\exp \left\{-\Lambda_{\mathrm{t}}^{4} \alpha^{2} \theta^{2}\right\}\right] / 2=\left[\exp \left\{-\mathrm{e}^{-2 \gamma \mathrm{t}} \times 2.5^{2}\right\}\right] / 2=\left[\exp \left\{-\mathrm{e}^{-2\left(\frac{2.5}{\alpha \times 0.0303}\right)} \times 2.5^{2}\right\}\right] / 2, \\
& \mathrm{P}_{\mathrm{err}}^{2 \text { nd }}=\mathrm{P}_{\mathrm{err}}^{3 \mathrm{rd}}=\left[\exp \left\{-\Lambda_{\mathrm{t}}^{6} \alpha^{2} \theta^{2}\right\}\right] / 2=\left[\exp \left\{-\mathrm{e}^{-3 \gamma \mathrm{t}} \times 2.5^{2}\right\}\right] / 2=\left[\exp \left\{-\mathrm{e}^{-3\left(\frac{2.5}{\alpha \times 0.0303}\right)} \times 2.5^{2}\right\}\right] / 2, \\
& \mathrm{P}_{\mathrm{err}}^{4 \text { th }}=\mathrm{P}_{\mathrm{err}}^{\mathrm{fin}}=\left[\exp \left\{-\Lambda_{\mathrm{t}}^{2} \alpha^{2} \theta^{2}\right\}\right] / 2=\left[\exp \left\{-\mathrm{e}^{-\gamma \mathrm{t}} \times 2.5^{2}\right\}\right] / 2=\left[\exp \left\{-\mathrm{e}^{-\left(\frac{2.5}{\alpha \times 0.0303}\right)} \times 2.5^{2}\right\}\right] / 2,
\end{aligned}
$$

where $\gamma t=2.5 /(\alpha \times 0.0303)$ for $\Lambda_{t}=e^{-\gamma t / 2}$ with a fixed $\alpha \theta=\alpha \chi t=2.5$, and a signal loss of $0.15 \mathrm{~dB} / \mathrm{km}$ $(\chi / \gamma=0.0303)$ in optical fibers ${ }^{73}$. From these calculations, we can obtain the efficiency values of the nonlinear optical gates under the decoherence effect. Figure 6 shows the tendencies of the error probabilities $\left(\mathrm{P}_{\mathrm{err}}^{1 s \mathrm{t}}, \mathrm{P}_{\mathrm{err}}^{2 \mathrm{nd}}\right.$, $\mathrm{P}_{\mathrm{err}}^{3 \mathrm{rd}}, \mathrm{P}_{\mathrm{err}}^{4 \mathrm{th}}$, and $\left.\mathrm{P}_{\mathrm{err}}^{\mathrm{fin}}\right)$ and rates $\left(\Lambda_{t}^{4}, \Lambda_{t}^{6}\right.$, and $\left.\Lambda_{t}^{2}\right)$ of the remaining photons of the gates (first, second, third, fourth, and final) in terms of the differences in the amplitude of the coherent state $(\alpha)$ with the following parameters: 


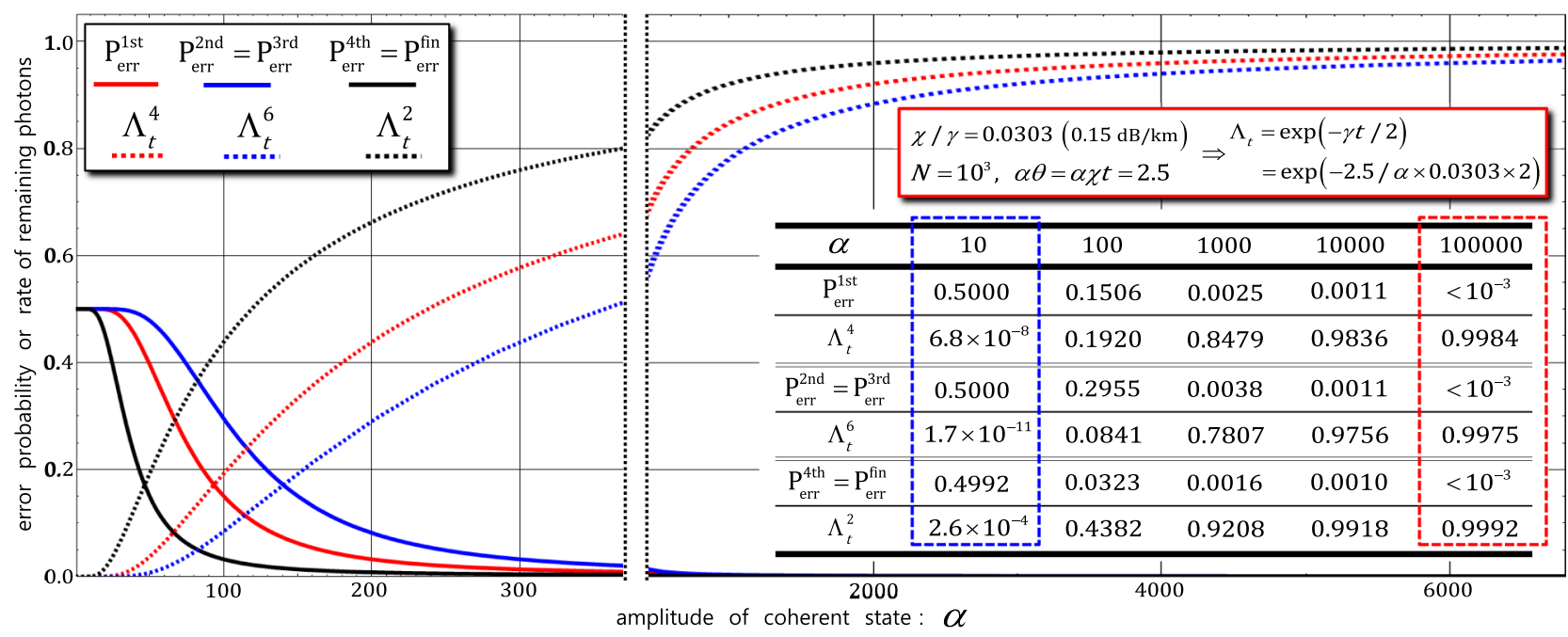

Figure 6. Error probabilities and rates of remaining photons in probe beam in practical case (under decoherence effect): Graph shows error probabilities $\left(\mathrm{P}_{\mathrm{err}}^{1 \mathrm{st}}, \mathrm{P}_{\mathrm{err}}^{2 \mathrm{nd}}, \mathrm{P}_{\mathrm{err}}^{3 \mathrm{rd}}, \mathrm{P}_{\mathrm{err}}^{4 \text { th }}\right.$, and $\left.\mathrm{P}_{\mathrm{err}}^{\mathrm{fin}}\right)$ and rates $\left(\Lambda_{t}=e^{-\gamma t / 2}\right)$ of remaining photons of nonlinear optical gates (first, second, third, fourth, and final) for differences in amplitude $(\alpha)$ of coherent state with fixed $\alpha \theta=2.5$ and signal loss of $0.15 \mathrm{~dB} / \mathrm{km}(\chi / \gamma=0.0303)$. Values of error probabilities and rates of remaining photons in each gate are listed in table.

signal loss of $0.15 \mathrm{~dB} / \mathrm{km}(\chi / \gamma=0.0303), \alpha \theta=\alpha \chi t=2.5$, and $N=10^{3}$. In addition, the values of error probabilities $\left(\mathrm{P}_{\text {err }}^{1 \text { st }}, \mathrm{P}_{\mathrm{err}}^{2 \text { nd }}, \mathrm{P}_{\mathrm{err}}^{3 \mathrm{rd}}, \mathrm{P}_{\mathrm{err}}^{4 \text { th }}\right.$, and $\left.\mathrm{P}_{\mathrm{err}}^{\mathrm{fin}}\right)$ and rates $\left(\Lambda_{t}^{4}, \Lambda_{t}^{6}\right.$, and $\left.\Lambda_{t}^{2}\right)$ of the remaining photons of the gates were calculated for the amplitudes, $\alpha=10,100,1000,10000$, and 100000, of the coherent state, as listed on Table (in Fig. 6). Compared with the dotted-blue box and dotted-red box in Table, we can conclude that high efficiencies, i.e., $\mathrm{P}_{\mathrm{err}}<10^{-3}$ (and low rate, $\Lambda_{t} \approx 1.0$ of photon loss), can be achieved in the nonlinear optical gates by employing a strong amplitude of coherent state $(\alpha \gg 10)$ under the decoherence effect.

Moreover, we should analyze the performances, the influence of dephasing, of the nonlinear optical gates, in addition to the efficiencies which are quantified by error probabilities by photon loss in Fig. 6 . To quantify the influences of dephasing of coherent parameters induced by the decoherence effect, we require a process model (Eqs. 13 and 14) that can describe the dynamics of the interactions of XKNLs $\left(\hat{X}_{t}\right)$ and the decoherence effect $\left(\hat{D}_{t}\right)$ to analyze the output states from the nonlinear optical gates.

In the first, fourth, and final gates, using the process model's formula (Eqs. 13 and 14), the output states $\left|\psi_{0}^{\prime}\right\rangle_{\mathrm{ABCD}}$ in Eq. (4), $\left|\psi_{3}^{\prime \prime}\right\rangle_{\mathrm{ABCD}}$ in Eq. (9), and $\left|\psi_{5}^{\prime}\right\rangle_{\mathrm{ABCD}}$ in Eq. (11) can be expressed as density matrices $\rho_{0}^{\prime}$ of the first gate, $\rho_{3}^{\prime \prime}$ of the fourth gate, and $\rho_{5}^{\prime}$ of the final gate, respectively, to determine the dephasing of coherent parameters, as follows:

$$
\rho_{0}^{\prime}=\frac{1}{4}\left(\begin{array}{cccc}
1 & |\mathrm{CK}|^{2} & |\mathrm{~L}|^{2} & |\mathrm{CO}|^{2} \\
|\mathrm{CK}|^{2} & 1 & |\mathrm{CO}|^{2} & |\mathrm{~L}|^{2} \\
|\mathrm{~L}|^{2} & |\mathrm{CO}|^{2} & 1 & |\mathrm{CM}|^{2} \\
|\mathrm{CO}|^{2} & |\mathrm{~L}|^{2} & |\mathrm{CM}|^{2} & 1
\end{array}\right), \quad \rho_{3}^{\prime \prime}=\rho_{5}^{\prime}=\frac{1}{2}\left(\begin{array}{cc}
1 & |\mathrm{C}|^{2} \\
|\mathrm{C}|^{2} & 1
\end{array}\right) \text {, }
$$

where the bases of $\rho_{0}^{\prime}$ are the states in $|L\rangle_{\mathrm{A}}^{1}|L\rangle_{\mathrm{B}}^{1}|R\rangle_{\mathrm{C}}^{1}|R\rangle_{\mathrm{D}}^{1}\left|\Lambda_{t}^{2} \alpha\right\rangle_{\mathrm{P}}^{\mathrm{a}}|0\rangle_{\mathrm{P}}^{\mathrm{b}},|L\rangle_{\mathrm{A}}^{1}|L\rangle_{\mathrm{B}}^{1}|R\rangle_{\mathrm{C}}^{2}|R\rangle_{\mathrm{D}}^{2}\left|\Lambda_{t}^{2} \alpha\right\rangle_{\mathrm{P}}^{\mathrm{a}}|0\rangle_{\mathrm{P}}^{\mathrm{b}}$, $|L\rangle_{\mathrm{A}}^{1}|L\rangle_{\mathrm{B}}^{1}|R\rangle_{\mathrm{C}}^{1}|R\rangle_{\mathrm{D}}^{2}\left|\Lambda_{t}^{2} \alpha \cos \theta\right\rangle_{\mathrm{P}}^{\mathrm{a}}\left|i \Lambda_{t}^{2} \alpha \sin \theta\right\rangle_{\mathrm{P}}^{\mathrm{b}}$, and $|L\rangle_{\mathrm{A}}^{1}|L\rangle_{\mathrm{B}}^{1}|R\rangle_{\mathrm{C}}^{1}|R\rangle_{\mathrm{D}}^{2}\left|\Lambda_{t}^{2} \alpha \cos \theta\right\rangle_{\mathrm{P}}^{\mathrm{a}}\left|-i \Lambda_{t}^{2} \alpha \sin \theta\right\rangle_{\mathrm{P}}^{\mathrm{b}}$; the bases of $\rho_{3}^{\prime \prime}$ are the states in $\left(\frac{1}{2}\left|0_{\mathrm{L}}\right\rangle_{\mathrm{ABCD}}^{1112}+\frac{\sqrt{3}}{2}\left|1_{\mathrm{L}}\right\rangle_{\mathrm{ABCD}}^{1111}\right)\left|\Lambda_{t} \alpha\right\rangle_{\mathrm{P}}^{\mathrm{a}}|0\rangle_{\mathrm{P}}^{\mathrm{b}}$ and $\left(\frac{1}{2}\left|0_{\mathrm{L}}\right\rangle_{\mathrm{ABCD}}^{1121}+\frac{\sqrt{3}}{2}\left|1_{\mathrm{L}}\right\rangle_{\mathrm{ABCD}}^{1122}\right)\left|\Lambda_{t} \alpha \cos \theta\right\rangle_{\mathrm{P}}^{\mathrm{a}}$ $\left|-i \Lambda_{t} \alpha \sin \theta\right\rangle_{\mathrm{P}}^{\mathrm{b}}$; the bases of $\rho_{5}^{\prime}$ are the states in $\left(\alpha_{1}\left|0_{\mathrm{L}}\right\rangle_{\mathrm{ABCD}}^{1111}+\beta_{1}\left|1_{\mathrm{L}}\right\rangle_{\mathrm{ABCD}}^{1111}\right)\left|\Lambda_{t} \alpha\right\rangle_{\mathrm{P}}^{\mathrm{a}}|0\rangle_{\mathrm{P}}^{\mathrm{b}}$ and $\left(\alpha_{2}\left|0_{\mathrm{L}}\right\rangle_{\mathrm{ABCD}}^{1112}-\beta_{2}\left|1_{\mathrm{L}}\right\rangle_{\mathrm{ABCD}}^{1112}\right)\left|\Lambda_{t} \alpha \cos \theta\right\rangle_{\mathrm{P}}^{\mathrm{a}}\left|-i \Lambda_{t} \alpha \sin \theta\right\rangle_{\mathrm{P}}^{\mathrm{b}}$ from left to right and top to bottom. Based on the process model expressed in Eq. (14), the coherent parameters in the density matrices $\left(\rho_{0}^{\prime}, \rho_{3}^{\prime \prime}\right.$, and $\left.\rho_{5}^{\prime}\right)$ are expressed as

$$
\mathrm{C}=\exp \left[-(\alpha / \sqrt{2})^{2}\left(1-\mathrm{e}^{-\gamma \Delta \mathrm{t}}\right) \sum_{\mathrm{n}=1}^{\mathrm{N}}\left(1-\mathrm{e}^{\mathrm{in} \Delta \theta}\right) \mathrm{e}^{-\gamma \Delta \mathrm{t}(\mathrm{n}-1)}\right],
$$




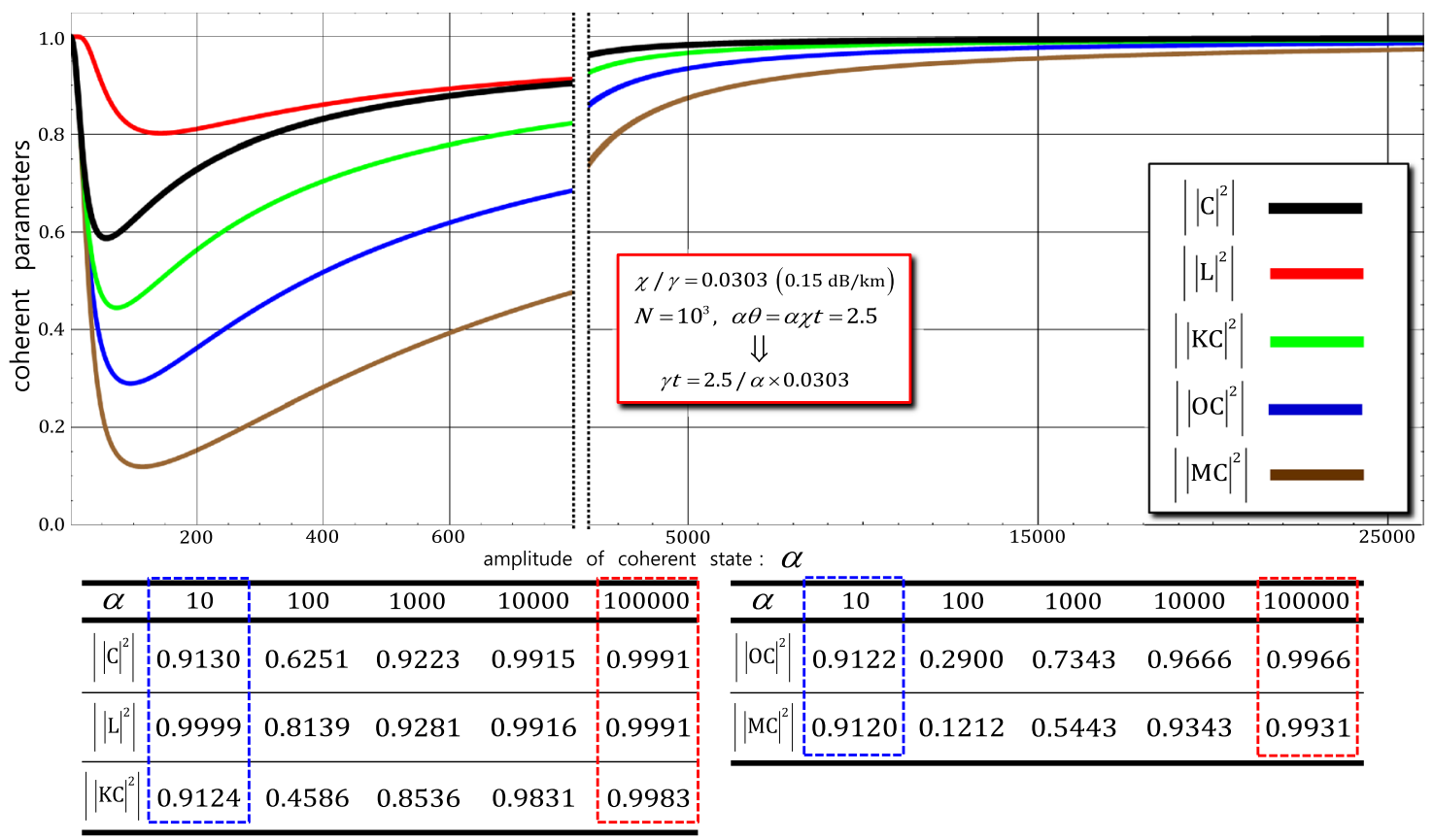

Figure 7. Trends and values of coherent parameters in output states of first, fourth, and final gates by dephasing (decoherence effect): Graph represents coherent parameters in output states of the nonlinear optical gates (first, fourth, and final) for differences in amplitude $(\alpha)$ of coherent state with signal loss of $0.15 \mathrm{~dB} / \mathrm{km}$ $(\chi / \gamma=0.0303), \alpha \theta=2.5\left(\mathrm{P}_{\mathrm{err}}<10^{-3}\right)$, and $N=10^{3}$. Values of coherent parameters in output states are listed in table.

$$
\begin{gathered}
\mathrm{L}=\exp \left[-(\alpha / \sqrt{2})^{2}\left(\mathrm{e}^{-\gamma \mathrm{t}}\right)\left(1-\mathrm{e}^{-\gamma \Delta \mathrm{t}}\right) \sum_{\mathrm{n}=1}^{\mathrm{N}}\left(1-\mathrm{e}^{\mathrm{in} \Delta \theta}\right) \mathrm{e}^{-\gamma \Delta \mathrm{t}(\mathrm{n}-1)}\right], \\
\mathrm{K}=\exp \left[-(\alpha / \sqrt{2})^{2}\left(\mathrm{e}^{-\gamma \mathrm{t}}\right)\left(1-\mathrm{e}^{-\gamma \Delta \mathrm{t}}\right) \sum_{\mathrm{n}=1}^{\mathrm{N}}\left(1-\mathrm{e}^{\mathrm{i} \theta} \cdot \mathrm{e}^{-\mathrm{in} \Delta \theta}\right) \mathrm{e}^{-\gamma \Delta \mathrm{t}(\mathrm{n}-1)}\right], \\
\mathrm{M}=\exp \left[-(\alpha / \sqrt{2})^{2}\left(\mathrm{e}^{-\gamma \mathrm{t}}\right)\left(1-\mathrm{e}^{-\gamma \Delta \mathrm{t}}\right) \sum_{\mathrm{n}=1}^{\mathrm{N}}\left(1-\mathrm{e}^{\mathrm{i} \theta} \cdot \mathrm{e}^{\mathrm{in} \Delta \theta}\right) \mathrm{e}^{-\gamma \Delta \mathrm{t}(\mathrm{n}-1)}\right], \\
\mathrm{O}=\exp \left[-(\alpha / \sqrt{2})^{2}\left(\mathrm{e}^{-\gamma \mathrm{t}}\right)\left(1-\mathrm{e}^{-\gamma \Delta \mathrm{t}}\right)\left(1-\mathrm{e}^{\mathrm{i} \theta}\right) \sum_{\mathrm{n}=1}^{\mathrm{N}} \mathrm{e}^{-\gamma \Delta \mathrm{t}(\mathrm{n}-1)}\right],
\end{gathered}
$$

where for $\theta=\chi t=\chi N \Delta t=N \Delta \theta$ and $\alpha \in \mathbb{R}$ with an arbitrarily small time $\Delta t=t / N$ (for a good approximation $\left.^{57-59,63,65}\right)$. In the density matrices $\left(\rho_{0}^{\prime}, \rho_{3}^{\prime \prime}\right.$, and $\left.\rho_{5}^{\prime}\right)$, the off-diagonal terms refer to the coherent parameters $(\mathrm{C}, \mathrm{L}, \mathrm{K}, \mathrm{M}$, and $\mathrm{O}$ ) that can be used to evaluate the degrees of a mixed state and quantify the influences of dephasing. For example, if the values of the coherent parameters (off-diagonal terms) decrease by dephasing (decoherence effect), then the output states ( $\rho_{0}^{\prime}$ of the first gate, $\rho_{3}^{\prime \prime}$ of the fourth gate, and $\rho_{5}^{\prime}$ of the final gate) evolve into mixed states, the ensemble of classical states. Therefore, to obtain reliable performances from the nonlinear optical gates, first, fourth, and final gates, the values of the coherent parameters should be retrained to approach 1 for the pure quantum state against dephasing by the decoherence effect. Figure 7 shows the tendencies of the coherent parameters $\left(\left.|| \mathrm{C}\right|^{2}|,||\mathrm{L}|^{2}|,||\mathrm{KC}|^{2}|,||\mathrm{OC}|^{2} \mid\right.$, and $\left.\left.|| \mathrm{MC}\right|^{2} \mid\right)$ in the density matrices $\left(\rho_{0}^{\prime}\right.$, $\rho_{3}^{\prime \prime}$, and $\rho_{5}^{\prime}$ ) of the first, fourth, and final gates for the amplitude of the coherent state, probe beam: $\alpha$, with the following parameters: signal loss of $0.15 \mathrm{~dB} / \mathrm{km}(\chi / \gamma=0.0303), \alpha \theta=\alpha \chi t=2.5\left(\mathrm{P}_{\mathrm{err}}<10^{-3}\right)$, and $N=10^{3}$ under the decoherence effect. In addition, based on the table shown in Fig. 7, we calculated the values of the coherent parameters based on the differences in the amplitudes $(\alpha=10,100,1000,10000$, and 100000) of the probe beams. As shown in Fig. 7, if the amplitude of the coherent state (probe beam) increases $(\alpha \gg 100)$, then all values of the coherent parameters are approximately 1 . Hence, by employing the strong (large amplitude) coherent state, we can maintain the output state $\left(\rho_{0}^{\prime}, \rho_{3}^{\prime \prime}\right.$, and $\left.\rho_{5}^{\prime}\right)$ of the first, fourth, and final gates to pure quantum states (prevention of off-diagonal terms in the density matrices, Eq. 16) against the influence of dephasing induced by the decoherence effect. Herein, as shown by the dotted blue box, the values of coherent parameters are high $(>0.9)$ when the amplitude of the coherent state is small $(\alpha=10)$, compared with the values within the dotted-red box in Fig. 7. However, in the small amplitude range $(\alpha<10)$ of the coherent state, we could not acquire a high rate of highly efficient remaining photons in the first, fourth, and final gates, as shown in Fig. 6 (dotted-blue box). 
Hence, for high efficiencies (low error probabilities) and reliable performances (preserved pure quantum states in the output) in the first, fourth, and final gates, we should utilize the strong coherent state (probe beam) to reduce the influences of photon loss and dephasing.

In the second and third gates, using the process model's formula (Eqs. 13 and 14), the output states $\left(\left|\psi_{1}^{\prime}\right\rangle{ }_{\mathrm{ABCD}}\right.$ in Eq. 5 and $\left|\psi_{2}^{\prime}\right\rangle \quad$ in Eq. 6) can be expressed as density matrices $\rho_{1}^{\prime}$ of the second gate and $\rho_{2}^{\prime}$ of the third gate, as follows:

$$
\rho_{1}^{\prime}=\rho_{2}^{\prime}=\frac{1}{8}\left(\begin{array}{cccccccc}
1 & |\mathrm{LN}|^{2} & \mathrm{C}^{*} \mathrm{~K}^{*} & \mathrm{C}^{*} \mathrm{LO}^{*}|\mathrm{~N}|^{2} & |\mathrm{P}|^{2} & \mathrm{C}^{*} \mathrm{~K}^{*}|\mathrm{P}|^{2} & |\mathrm{LR}|^{2} & \mathrm{C}^{*} \mathrm{LO}^{*}|\mathrm{R}|^{2} \\
|\mathrm{LN}|^{2} & 1 & \mathrm{C}^{*} \mathrm{LO}^{*}|\mathrm{~N}|^{2} & \mathrm{C}^{*} \mathrm{~K}^{*} & |\mathrm{LR}|^{2} & \mathrm{C}^{*} \mathrm{LO}^{*}|\mathrm{R}|^{2} & |\mathrm{P}|^{2} & \mathrm{C}^{*} \mathrm{~K}^{*}|\mathrm{P}|^{2} \\
\mathrm{CK} & \mathrm{CL}^{*} \mathrm{O}|\mathrm{N}|^{2} & 1 & |\mathrm{CON}|^{2} & \mathrm{CK}|\mathrm{P}|^{2} & |\mathrm{P}|^{2} & \mathrm{CL} \mathrm{O}|\mathrm{R}|^{2} & |\mathrm{COR}|^{2} \\
\mathrm{CL}^{*} \mathrm{O}|\mathrm{N}|^{2} & \mathrm{CK} & |\mathrm{CON}|^{2} & 1 & \mathrm{CL}^{*} \mathrm{O}|\mathrm{R}|^{2} & |\mathrm{COR}|^{2} & \mathrm{CK}|\mathrm{P}|^{2} & |\mathrm{P}|^{2} \\
|\mathrm{P}|^{2} & |\mathrm{LR}|^{2} & \mathrm{C}^{*} \mathrm{~K}^{*}|\mathrm{P}|^{2} & \mathrm{C}^{*} \mathrm{LO}^{*}|\mathrm{R}|^{2} & 1 & \mathrm{CK} & |\mathrm{LS}|^{2} & \mathrm{C}^{*} \mathrm{LO}^{*}|\mathrm{~S}|^{2} \\
\mathrm{CK}|\mathrm{P}|^{2} & \mathrm{CL}^{*} \mathrm{O}|\mathrm{R}|^{2} & |\mathrm{P}|^{2} & |\mathrm{COR}|^{2} & \mathrm{C}^{*} \mathrm{~K}^{*} & 1 & \mathrm{CL}{ }^{*} \mathrm{O}||^{2} & |\mathrm{COS}|^{2} \\
|\mathrm{LR}|^{2} & |\mathrm{P}|^{2} & \mathrm{C}^{*} \mathrm{LO}^{*}|\mathrm{R}|^{2} & \mathrm{C}^{*} \mathrm{~K}^{*}|\mathrm{P}|^{2} & |\mathrm{LS}|^{2} & \mathrm{C}^{*} \mathrm{LO}^{*}|\mathrm{~S}|^{2} & 1 & \mathrm{C}^{*} \mathrm{~K}^{*} \\
\mathrm{CL} \mathrm{C}^{*} \mathrm{O}||^{2} & \mathrm{CK}|\mathrm{P}|^{2} & |\mathrm{COR}|^{2} & |\mathrm{P}|^{2} & \mathrm{CL}^{*} \mathrm{O}|\mathrm{S}|^{2} & |\mathrm{COS}|^{2} & \mathrm{CK} & 1
\end{array}\right)
$$

where the bases of $\rho_{1}^{\prime}$ are the states in $|H\rangle_{\mathrm{A}}^{1}|L\rangle_{\mathrm{B}}^{1}|V\rangle_{\mathrm{C}}^{1}|R\rangle_{\mathrm{D}}^{1}\left|\Lambda_{t}^{3} \alpha\right\rangle_{\mathrm{P}}^{\mathrm{a}}|0\rangle_{\mathrm{P}}^{\mathrm{b}},|H\rangle_{\mathrm{A}}^{1}|L\rangle_{\mathrm{B}}^{1}|R\rangle_{\mathrm{C}}^{2}|V\rangle_{\mathrm{D}}^{2}\left|\Lambda_{t}^{3} \alpha\right\rangle_{\mathrm{P}}^{\mathrm{a}}|0\rangle_{\mathrm{P}}^{\mathrm{b}}$, $|V\rangle_{\mathrm{A}}^{1}|L\rangle_{\mathrm{B}}^{1}|H\rangle_{\mathrm{C}}^{1}|R\rangle_{\mathrm{D}}^{1}\left|\Lambda_{t}^{3} \alpha\right\rangle_{\mathrm{P}}^{\mathrm{a}}|0\rangle_{\mathrm{P}}^{\mathrm{b}},|V\rangle_{\mathrm{A}}^{1}|L\rangle_{\mathrm{B}}^{1}|R\rangle_{\mathrm{C}}^{1}|H\rangle_{\mathrm{D}}^{1}\left|\Lambda_{t}^{3} \alpha\right\rangle_{\mathrm{P}}^{\mathrm{a}}|0\rangle_{\mathrm{P}}^{\mathrm{b}}|H\rangle_{\mathrm{A}}^{1}|L\rangle_{\mathrm{B}}^{1}|H\rangle_{\mathrm{C}}^{1}|R\rangle_{\mathrm{D}}^{1}\left|\Lambda_{t}^{3} \alpha \cos \theta\right\rangle_{\mathrm{P}}^{\mathrm{a}}\left|i \Lambda_{t}^{3} \alpha \sin \theta\right\rangle_{\mathrm{P}}^{\mathrm{b}}$, $|H\rangle_{\mathrm{A}}^{1}|L\rangle_{\mathrm{B}}^{1}|R\rangle_{\mathrm{C}}^{2}|H\rangle_{\mathrm{D}}^{2}\left|\Lambda_{t}^{3} \alpha \cos \theta\right\rangle_{\mathrm{P}}^{\mathrm{a}}\left|i \Lambda_{t}^{3} \alpha \sin \theta\right\rangle_{\mathrm{P}}^{\mathrm{b}},|V\rangle_{\mathrm{A}}^{1}|L\rangle_{\mathrm{B}}^{1}|V\rangle_{\mathrm{C}}^{1}|R\rangle_{\mathrm{D}}^{1}\left|\Lambda_{t}^{3} \alpha \cos \theta\right\rangle_{\mathrm{P}}^{\mathrm{a}}\left|-i \Lambda_{t}^{3} \alpha \sin \theta\right\rangle_{\mathrm{P}}^{\mathrm{b}}$, and $|V\rangle_{\mathrm{A}}^{1}|L\rangle_{\mathrm{B}}^{1}$ $|R\rangle_{\mathrm{C}}^{2}|V\rangle_{\mathrm{D}}^{2}\left|\Lambda_{t}^{3} \alpha \cos \theta\right\rangle_{\mathrm{P}}^{\mathrm{a}}\left|-i \Lambda_{t}^{3} \alpha \sin \theta\right\rangle_{\mathrm{P}}^{\mathrm{b}}$; the bases of $\rho_{2}^{\prime}$ are the states in $\frac{1}{\sqrt{2}}|H\rangle_{\mathrm{A}}^{1}|H\rangle_{\mathrm{B}}^{1}\left(|V\rangle_{\mathrm{C}}^{1}|V\rangle_{\mathrm{D}}^{1}+|V\rangle_{\mathrm{C}}^{2}|V\rangle_{\mathrm{D}}^{2}\right)$ $\left|\Lambda_{t}^{3} \alpha\right\rangle_{\mathrm{P}}^{\mathrm{a}}|0\rangle_{\mathrm{P}}^{\mathrm{b}}, \frac{1}{\sqrt{2}}|V\rangle_{\mathrm{A}}^{1}|H\rangle_{\mathrm{B}}^{1}\left(|H\rangle_{\mathrm{C}}^{1}|V\rangle_{\mathrm{D}}^{1}+|V\rangle_{\mathrm{C}}^{2}|H\rangle_{\mathrm{D}}^{2}\right)\left|\Lambda_{t}^{3} \alpha\right\rangle_{\mathrm{P}}^{\mathrm{a}}|0\rangle_{\mathrm{P}}^{\mathrm{b}}, \frac{1}{\sqrt{2}}|V\rangle_{\mathrm{A}}^{1}|V\rangle_{\mathrm{B}}^{1}\left(|H\rangle_{\mathrm{C}}^{1}|H\rangle_{\mathrm{D}}^{1}+|H\rangle_{\mathrm{C}}^{2}|H\rangle_{\mathrm{D}}^{2}\right)\left|\Lambda_{t}^{3} \alpha\right\rangle_{\mathrm{P}}^{\mathrm{a}}|0\rangle_{\mathrm{P}}^{\mathrm{b}}$, $\frac{1}{\sqrt{2}}|H\rangle_{\mathrm{A}}^{1}|V\rangle_{\mathrm{B}}^{1}\left(|H\rangle_{\mathrm{C}}^{2}|V\rangle_{\mathrm{D}}^{2}+|V\rangle_{\mathrm{C}}^{1}|H\rangle_{\mathrm{D}}^{1}\right)\left|\Lambda_{t}^{3} \alpha\right\rangle_{\mathrm{P}}^{\mathrm{a}}|0\rangle_{\mathrm{P}}^{\mathrm{b}}, \frac{1}{\sqrt{2}}|H\rangle_{\mathrm{A}}^{1}|H\rangle_{\mathrm{B}}^{1}\left(|V\rangle_{\mathrm{C}}^{1}|H\rangle_{\mathrm{D}}^{1}+|H\rangle_{\mathrm{C}}^{2}|V\rangle_{\mathrm{D}}^{2}\right)\left|\Lambda_{t}^{3} \alpha \cos \theta\right\rangle_{\mathrm{P}}^{\mathrm{a}}\left|i \Lambda_{t}^{3} \alpha \sin \theta\right\rangle_{\mathrm{P}}^{\mathrm{b}}$, $\frac{1}{\sqrt{2}}|V\rangle_{\mathrm{A}}^{1}|H\rangle_{\mathrm{B}}^{1}\left(|H\rangle_{\mathrm{C}}^{1}|H\rangle_{\mathrm{D}}^{1}+|H\rangle_{\mathrm{C}}^{2}|H\rangle_{\mathrm{D}}^{2}\right)\left|\Lambda_{t}^{3} \alpha \cos \theta\right\rangle_{\mathrm{P}}^{\mathrm{a}}\left|i \Lambda_{t}^{3} \alpha \sin \theta\right\rangle_{\mathrm{P}}^{\mathrm{b}}, \quad \frac{1}{\sqrt{2}}|V\rangle_{\mathrm{A}}^{1}|V\rangle_{\mathrm{B}}^{1}\left(|H\rangle_{\mathrm{C}}^{1}|V\rangle_{\mathrm{D}}^{1}+|V\rangle_{\mathrm{C}}^{2}|H\rangle_{\mathrm{D}}^{2}\right)$ $\left|\Lambda_{t}^{3} \alpha \cos \theta\right\rangle_{\mathrm{P}}^{\mathrm{a}}\left|-i \Lambda_{t}^{3} \alpha \sin \theta\right\rangle_{\mathrm{P}}^{\mathrm{b}}$, and $\frac{1}{\sqrt{2}}|H\rangle_{\mathrm{A}}^{1}|V\rangle_{\mathrm{B}}^{1}\left(|V\rangle_{\mathrm{C}}^{1}|V\rangle_{\mathrm{D}}^{1}+|V\rangle_{\mathrm{C}}^{2}|V\rangle_{\mathrm{D}}^{2}\right)\left|\Lambda_{t}^{3} \alpha \cos \theta\right\rangle_{\mathrm{P}}^{\mathrm{a}}\left|-i \Lambda_{t}^{3} \alpha \sin \theta\right\rangle_{\mathrm{P}}^{\mathrm{b}}$ from left to right and top to bottom. The coherent parameters ( $, \mathrm{L}, \mathrm{K}, \mathrm{M}$, and $\mathrm{O}$ ) can be calculated using Eq. (17). The other coherent parameters $(\mathrm{P}, \mathrm{N}, \mathrm{S}$, and $\mathrm{R})$ in density matrices $\left(\rho_{1}^{\prime}\right.$ and $\left.\rho_{2}^{\prime}\right)$ can be calculated from the process model (Eq. 14), as follows:

$$
\begin{gathered}
\mathrm{P}=\exp \left[-(\alpha / \sqrt{2})^{2}\left(\mathrm{e}^{-2 \gamma \mathrm{t}}\right)\left(1-\mathrm{e}^{-\gamma \Delta \mathrm{t}}\right) \sum_{\mathrm{n}=1}^{\mathrm{N}}\left(1-\mathrm{e}^{\mathrm{in} \Delta \theta}\right) \mathrm{e}^{-\gamma \Delta \mathrm{t}(\mathrm{n}-1)}\right], \\
\mathrm{N}=\exp \left[-(\alpha / \sqrt{2})^{2}\left(\mathrm{e}^{-2 \gamma \mathrm{t}}\right)\left(1-\mathrm{e}^{-\gamma \Delta \mathrm{t}}\right) \sum_{\mathrm{n}=1}^{\mathrm{N}}\left(1-\mathrm{e}^{\mathrm{i} \theta} \cdot \mathrm{e}^{-\mathrm{in} \Delta \theta}\right) \mathrm{e}^{-\gamma \Delta \mathrm{t}(\mathrm{n}-1)}\right], \\
\mathrm{S}=\exp \left[-(\alpha / \sqrt{2})^{2}\left(\mathrm{e}^{-2 \gamma \mathrm{t}}\right)\left(1-\mathrm{e}^{-\gamma \Delta \mathrm{t}}\right) \sum_{\mathrm{n}=1}^{\mathrm{N}}\left(1-\mathrm{e}^{\mathrm{i} \theta} \cdot \mathrm{e}^{\mathrm{in} \Delta \theta}\right) \mathrm{e}^{-\gamma \Delta \mathrm{t}(\mathrm{n}-1)}\right], \\
\mathrm{R}=\exp \left[-(\alpha / \sqrt{2})^{2}\left(\mathrm{e}^{-2 \gamma \mathrm{t}}\right)\left(1-\mathrm{e}^{-\gamma \Delta \mathrm{t}}\right)\left(1-\mathrm{e}^{\mathrm{i} \theta}\right) \sum_{\mathrm{n}=1}^{\mathrm{N}} \mathrm{e}^{-\gamma \Delta \mathrm{t}(\mathrm{n}-1)}\right],
\end{gathered}
$$

where $\theta=\chi t=\chi N \Delta t=N \Delta \theta$ for an arbitrarily small time $\Delta t(=t / N)$ with $\alpha \in \mathbb{R}$. For the experimental condition to preserve the values of coherent parameters to 1 (pure quantum states) under the decoherence effect, we can determine the tendencies of the coherent parameters, off-diagonal terms, in the density matrices $\left(\rho_{1}^{\prime}\right.$ and $\rho_{2}^{\prime}$ ) of the second and third gates for the amplitude of the coherent state (probe beam: $\alpha$ ) using the following parameters: signal loss of $0.15 \mathrm{~dB} / \mathrm{km}(\chi / \gamma=0.0303), \alpha \theta=\alpha \chi t=2.5\left(\mathrm{P}_{\text {err }}<10^{-3}\right)$, and $N=10^{3}$, as shown in Fig. 8. In addition, the values of the coherent parameters are listed on Table (in Fig. 8) based on the differences in the amplitudes, $\alpha=10,100,1000,10000$, and 100000, of the probe beams. When the amplitude of the coherent state (probe beam) increased $(\alpha \gg 100$ ), all values of the coherent parameters were approximately 1 , similar to the coherent parameters in $\rho_{0}^{\prime}, \rho_{3}^{\prime \prime}$, and $\rho_{5}^{\prime}$, as shown in Fig. 8. Therefore, we confirmed that the influences, which evolved to mixed states, of dephasing coherent parameters in $\rho_{1}^{\prime}$ and $\rho_{2}^{\prime}$ (Eq. 18) can be reduced using a strong coherent state. Furthermore, by comparing with the dotted-blue box and dotted-red box in Fig. 8, the nonlinear optical gates (second and third) can yield high efficiencies (low error probabilities) and reliable performances (preserving pure quantum states) in the large amplitude range $(\alpha>100000)$ of the coherent state.

According to our analysis, using the process model (Eq. 14), which can be used to formulate the interaction of XKNLs between a signal system (photon) and a probe beam (coherent state) via the master equation (Eq. 13), we can conclude that the only experimental condition is to utilize the strong coherent state (probe beam) to reduce the influences of photon loss and dephasing (decoherence effect). Hence, we can obtain high efficiencies (low error probabilities, Fig. 6) and reliable performances (values of coherent parameters approaching 1: Figs. 7 and 8 ) in the nonlinear optical gates. Consequently, the proposed procedure for generating single logical qubit information (quantum information on four-photon decoherence-free states) with immunity against collective 

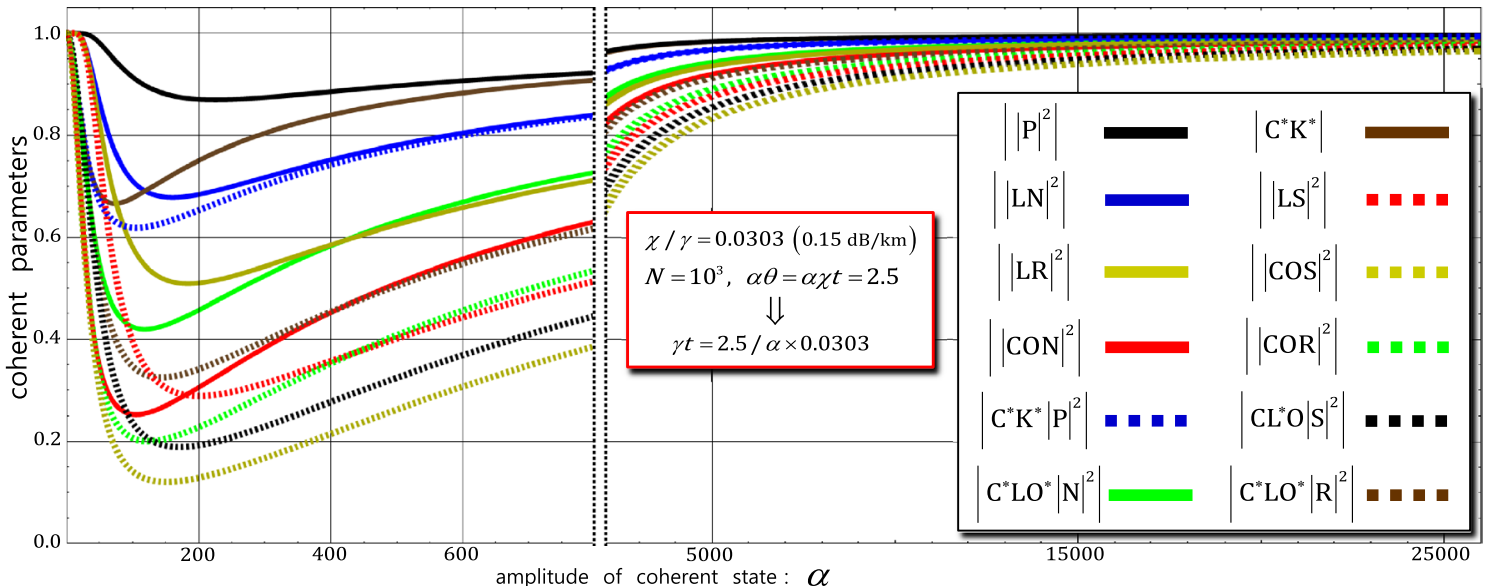

\begin{tabular}{c|c|ccc|c|}
\hline$\alpha$ & 10 & 100 & 1000 & 10000 & 100000 \\
\hline$\left.|| \mathrm{P}\right|^{2} \mid$ & 0.9999 & 0.9137 & 0.9337 & 0.9916 & 0.9991 \\
\hline$\left.|| \mathrm{LN}\right|^{2} \mid$ & 0.9999 & 0.7106 & 0.8643 & 0.9832 & 0.9983 \\
\hline$\left.|| \mathrm{LR}\right|^{2} \mid$ & 0.9999 & 0.5810 & 0.7525 & 0.9668 & 0.9966 \\
\hline$\left.|| \mathrm{CON}\right|^{2}$ & 0.9122 & 0.2529 & 0.6838 & 0.9584 & 0.9957 \\
\hline$\left.\left|\mathrm{C}^{*} \mathrm{~K}^{*}\right| \mathrm{P}\right|^{2}$ & 0.9552 & 0.6188 & 0.8626 & 0.9832 & 0.9983 \\
\hline$\left.\left|\mathrm{C}^{*} \mathrm{LO}{ }^{*}\right| \mathrm{N}\right|^{2}$ & 0.9551 & 0.4239 & 0.7688 & 0.9708 & 0.9970 \\
\hline
\end{tabular}

\begin{tabular}{c|c|ccc|c|}
\hline$\alpha$ & 10 & 100 & 1000 & 10000 & 100000 \\
\hline$\left|\mathrm{C}^{*} \mathrm{~K}^{*}\right|$ & 0.9552 & 0.6772 & 0.9239 & 0.9915 & 0.9991 \\
\hline$\left.|| \mathrm{LS}\right|^{2} \mid$ & 0.9999 & 0.3966 & 0.5711 & 0.9348 & 0.9932 \\
\hline$\left.|| \mathrm{CoS}\right|^{2} \mid$ & 0.9122 & 0.1411 & 0.4518 & 0.9112 & 0.9906 \\
\hline$\left.|| \mathrm{COR}\right|^{2} \mid$ & 0.9122 & 0.2068 & 0.5953 & 0.9424 & 0.9940 \\
\hline$\left.\left|\mathrm{CL}^{*} \mathrm{O}\right| \mathrm{S}\right|^{2} \mid$ & 0.9551 & 0.2366 & 0.5080 & 0.9230 & 0.9919 \\
\hline$\left.\left|\mathrm{C}^{*} \mathrm{LO} 0^{*}\right| \mathrm{R}\right|^{2} \mid$ & 0.9551 & 0.3466 & 0.6693 & 0.9546 & 0.9953 \\
\hline
\end{tabular}

Figure 8. Trends and values of coherent parameters in output states of second and third gates by dephasing (decoherence effect): Graph represents coherent parameters in output states of nonlinear optical gates (second and third) for differences in amplitude $(\alpha)$ of coherent state with signal loss of $0.15 \mathrm{~dB} / \mathrm{km}(\chi / \gamma=0.0303)$, $\alpha \theta=2.5\left(\mathrm{P}_{\mathrm{err}}<10^{-3}\right)$, and $N=10^{3}$. Values of coherent parameters in output states are listed in table.

decoherence can be experimentally implemented and is secure against photon loss and dephasing induced by the decoherence effect.

\section{Conclusion}

We proposed a procedure (Fig. 2) that can generate four-photon decoherence-free states, logical qubits, and encode quantum information onto the superposition of logical qubits (single logical qubit information) using XKNLs and linear optical devices to secure quantum information against collective decoherence. In addition, in the nonlinear optical gates, first, second, third, fourth, and final gates, we analyzed the influences of photon loss and dephasing induced by the decoherence effect, and then demonstrated the experimental condition to obtain high efficiencies and reliable performances for the feasible procedure (Fig. 2). The advantages of our procedure are as follows:

(1) Our procedure can be used to encode single logical qubit information and secure quantum information from collective decoherence using arbitrary information encoded onto the superposed state of four-photon decoherence-free states. The previous works, which proposed three-qubit decoherence-free states ${ }^{37-41}, \mathrm{can}$ provide the limited effect for the prevention against the affections of collective decoherence. Here, compared with three-qubit systems ${ }^{37-41}$, our scheme can generate four-qubit systems (four-photon decoherence-free states), which are more immune against collective decoherence, to maintain the coherence of quantum information.

(2) In various quantum information processing schemes, including the procedure of generating decoherencefree states, the noises, photon loss and dephasing, induced by the decoherence effect is inevitable. Thus, the method to reduce the influences of decoherence effect should be proposed. Before the works ${ }^{42,43}$ using cavity-QED system for four-qubit decoherence-free states, their schemes overlooked the affections of decoherence effect. Here, in our scheme, we discussed the quantifications of the influences of photon loss and dephasing from the decoherence effect. Accordingly, we demonstrated the experimental condition by our analysis using master equation to utilize a strong, large amplitude, coherent state for the high efficiencies, low error probabilities, and reliable performances by preserving pure quantum state in nonlinear optical gates under the decoherence effect. Thus, our scheme is experimentally more feasible, compared with the previous works ${ }^{42,43}$. 
(3) In our procedure, the nonlinear optical gates utilized only the positive conditional phase shifts $(\theta)$ of the XKNLs with qubus beams and PNR measurements because it is generally not possible to change the sign of the conditional phase shift $(-\theta)$ by $\mathrm{Kok}^{76}$. Moreover, the usage of a strong coherent state (for high efficiency and reliable performance) can yield an experimental advantage from using the weak conditional phase shift magnitude of the XKNL (i.e., if we use $\alpha=10^{5}$ to reduce the influence of the decoherence effect, then the XKNL magnitude required is $\theta=2.5 \times 10^{-5}$ for a fixed $\alpha \theta=2.5$ and $\left.\mathrm{P}_{\mathrm{err}}<10^{-3}\right)$. Hence, our nonlinear optical gates are more feasible in practice, as realizing a large conditional phase shift magnitude via the XKNL is difficult (extremely weak: $\left.\theta \approx 10^{-18}\right)^{77-79}$.

Consequently, our procedure, which can generate four-photon decoherence-free states, logical qubits, and encode quantum information onto the superposition of logical qubits (single logical qubit information), was designed to prevent quantum information from collective decoherence. In addition, for the feasible procedure (Fig. 2), we demonstrated that the nonlinear optical gates (first, second, third, fourth, and final) can yield high efficiencies, obtaining low error probabilities, and reliable performances, preserving pure quantum state, against photon loss and dephasing of the decoherence effect when a strong coherent state, probe beam, was used.

Received: 4 March 2021; Accepted: 30 April 2021

Published online: 17 May 2021

\section{References}

1. Bennett, C. H. et al. Teleporting an unknown quantum state via dual classical and Einstein-Podolsky-Rosen channels. Phys. Rev. Lett. 70, 1895 (1993).

2. Bouwmeester, D. et al. Experimental quantum teleportation. Nature 390, 575 (1997).

3. Bostrom, K. \& Felbinger, F. Deterministic secure direct communication using entanglement. Phys. Rev. Lett. 89, 187902 (2002).

4. Zeng, G. H. \& Keitel, C. H. Arbitrated quantum-signature scheme. Phys. Rev. A 65, 042312 (2002).

5. Tan, X. \& Zhang, X. Controlled quantum secure direct communication by entanglement distillation or generalized measurement. Quantum Inf. Process. 15, 2137 (2016).

6. Heo, J., Hong, C., Kang, M. S. \& Yang, H. J. Scheme for bidirectional quantum teleportation of unknown electron-spin states of quantum dots within single-sided cavities. Int. J. Theor. Phys. 59, 3705 (2020).

7. Du, H., Malaney, R. \& Green, J. Satellite-based distribution of hybrid entanglement. Quant. Eng. 3, e60 (2021).

8. Wang, C. Quantum secure direct communication: Intersection of communication and cryptography. Fundam. Res. 1, 91 (2021).

9. Deng, F. G., Ren, B. C. \& Li, X. H. Quantum hyperentanglement and its applications in quantum information processing. Sci. Bull. 62, 46 (2017).

10. Heo, J. et al. Distribution of hybrid entanglement and hyperentanglement with time-bin for secure quantum channel under noise via weak cross-Kerr nonlinearity. Sci. Rep. 7, 10208 (2017).

11. Steinlechner, F. et al. Distribution of high-dimensional entanglement via an intra-city free-space link. Nat. Commun. 8, 15971 (2017).

12. Heo, J., Hong, C., Choi, S. G. \& Hong, J. P. Scheme for generation of three-photon entangled $W$ state assisted by cross-Kerr nonlinearity and quantum dot. Sci. Rep. 9, 10151 (2019).

13. Zhang, Z., Scully, M. O. \& Agarwal, G. S. Quantum entanglement between two magnon modes via Kerr nonlinearity driven far from equilibrium. Phys. Rev. Res. 1, 023021 (2019).

14. Hong, C. et al. Generation of two-photon hybrid-entangled $W$ state with photonic qubit and time-bin via cross-Kerr nonlinearities. Phys. Scr. 95, 085104 (2020).

15. Sheng, Y. B. \& Zhou, L. Distributed secure quantum machine learning. Sci. Bull. 62, 1025-1029 (2017).

16. Kang, M. S., Heo, J., Choi, S. G., Moon, S. \& Han, S. W. Implementation of SWAP test for two unknown states in photons via cross-Kerr nonlinearities under decoherence effect. Sci. Rep. 9, 6167 (2019).

17. Bouten, L., Vissers, G. \& Schmidt-Kaler, F. “Quantum algorithm for simulating an experiment: Light interference from single ions and their mirror images. Phys. Rev. A 100, 022323 (2019).

18. Hong, C. et al. Photonic scheme of quantum phase estimation for quantum algorithms via cross-Kerr nonlinearities under decoherence effect. Opt. Express 27, 31023 (2019).

19. Heo, J., Won, K., Yang, H. J., Hong, J. P. \& Choi, S. G. Photonic scheme of discrete quantum Fourier transform for quantum algorithms via quantum dots. Sci. Rep. 9, 12440 (2019).

20. Kang, M. S., Heo, J., Choi, S. G., Sung, M. \& Han, S. W. Optical Fredkin gate assisted by quantum dot within optical cavity under vacuum noise and sideband leakage. Sci. Rep. 10, 1-13 (2020).

21. Li, K., Yan, P. G. \& Cai, Q. Y. Quantum computing and the security of public key cryptography. Fundam. Res. 1, 85 (2021).

22. Quan, J. Y., Li, Q., Liu, C. D., Shi, J. J. \& Peng, Y. A simplified verifiable blind quantum computing protocol with quantum input verification. Quant. Eng. 3, e58 (2021).

23. Shor, P. W. Scheme for reducing decoherence in quantum computer memory. Phys. Rev. A 52, R2493 (1995).

24. Steane, A. Multiple-particle interference and quantum error correction. Proc. R. Soc. A 452, 2551 (1996).

25. Lidar, D. \& Brun, T. Quantum Error Correction (Cambridge University Press, 2013).

26. Hu, X. M. et al. Long-distance entanglement purification for quantum communication. Phys. Rev. Lett. 126, 010503 (2021)

27. Yan, P. S., Zhou, L., Zhong, W. \& Sheng, Y. B. Feasible time-bin entanglement purification based on sum-frequency generation. Opt. Express 29, 571 (2021).

28. Yan, P. S., Zhou, L., Zhong, W. \& Sheng, Y. B. Feasible measurement-based entanglement purification in linear optics. Opt. Express 29, 9363 (2021).

29. Sheng, Y. B. et al. Efficient single-photon-assisted entanglement concentration for partially entangled photon pairs. Phys. Rev. A 85, 012307 (2012).

30. Sheng, Y. B. et al. Efficient two-step entanglement concentration for arbitrary W states. Phys. Rev. A 85, 042302 (2012).

31. Sheng, Y. B. et al. Efficient N-particle W state concentration with different parity check gates. Sci. China Phys. Mech. Astron. 58, 060301 (2015).

32. Zanardi, P. \& Rasetti, M. Noiseless quantum codes. Phys. Rev. Lett. 79, 3306 (1997).

33. Kempe, J., Bacon, D., Lidar, D. A. \& Whaley, K. B. Theory of decoherence-free fault-tolerant universal quantum computation. Phys. Rev. A 63, 042307 (2001).

34. Viola, L. et al. Experimental realization of noiseless subsystems for quantum information processing. Science 293, 2059 (2001).

35. Viola, L., Knill, E. \& Laflamme, R. Constructing qubits in physical systems. J. Phys. A 34, 7067 (2001). 
36. Altepeter, J. B., Hadley, P. G., Wendelken, S. M., Berglund, A. J. \& Kwiat, P. G. Experimental investigation of a two-qubit decoherence-free subspace. Phys. Rev. Lett. 92, 147901 (2004).

37. Dong, L. et al. Single logical qubit information encoding scheme with the minimal optical decoherence-free subsystem. Opt. Lett. 41, 1030 (2016).

38. Dong, L. et al. Generation of three-photon polarization-entangled decoherence-free states. Ann. Phys. 371, 287 (2016).

39. Heo, J., Kang, M. S., Hong, C. H., Hong, J. P. \& Choi, S. G. Preparation of quantum information encoded on three-photon decoherence-free states via cross-Kerr nonlinearities. Sci. Rep. 8, 13843 (2018).

40. Hong, C., Heo, J., Kang, M. S., Jang, J. \& Yang, H. J. Scheme for encoding single logical qubit information into three-photon decoherence-free states assisted by quantum dots. Quantum Inf. Process. 18, 216 (2019).

41. Shao, X. Q., Chen, L., Zhang, S., Zhao, Y. F. \& Yeon, K. H. Preparation of three- and four-qubit decoherence-free states via Zenolike measurements. J. Phys. B 43, 135502 (2010).

42. Chen, C. Photonic four-qubit entangled decoherence-free states assisted by cavity-QED system. Int. J Theor. Phys. 55, 4841 (2016).

43. Zhou, Y. S., Li, X., Deng, Y., Li, H. R. \& Luo, M. X. Generation of hybrid four-qubit entangled decoherence-free states assisted by the cavity-QED system. Opt. Commun. 366, 397 (2016).

44. Li, Y. B., Song, T. T., Huang, W. \& Zhan, W. W. Fault-tolerant quantum secure direct communication protocol based on decoherence-free states. Int. J Theor. Phys. 54, 589 (2015).

45. Wang, M., Yan, F. \& Gao, T. Generation of four-photon polarization entangled decoherence-free states with cross-Kerr nonlinearity. Sci. Rep. 6, 38233 (2016).

46. Xiu, X. M. et al. Preparation of four-photon polarization-entangled decoherence-free states employing weak cross-Kerr nonlinearities. Phys. Rev. A 94, 042321 (2016).

47. Wang, M., Yan, F. \& Gao, T. Generation of an arbitrary four-photon polarization-entangled decoherence-free state with cross-Kerr nonlinearity. Quantum Inf. Process. 16, 195 (2017).

48. Heo, J., Hong, C., Kang, M. S. \& Yang, H. J. Encoding scheme using quantum dots for single logical qubit information onto fourphoton decoherence-free states. Sci. Rep. 10, 15334 (2020).

49. Agrawal, P. \& Pati, A. Perfect teleportation and superdense coding with W states. Phys. Rev. A 74, 062320 (2006).

50. Chen, J., Zhou, H., Duan, C. \& Peng, X. Preparing Greenberger-Horne-Zeilinger and W states on a long-range Ising spin model by global controls. Phys. Rev. A 95, 032340 (2017).

51. Dur, W., Vidal, G. \& Cirac, J. I. Three qubits can be entangled in two inequivalent ways. Phys. Rev. A 62, 062314 (2000).

52. Zou, X. B., Shu, J. \& Guo, G. C. Simple scheme for generating four-photon polarization-entangled decoherence-free states using spontaneous parametric down-conversions. Phys. Rev. A 73, 054301 (2006).

53. Gong, Y. X. et al. Generation of arbitrary four-photon polarization-entangled decoherence-free states. Phys. Rev. A 77, 042317 (2008).

54. Xia, Y., Song, J., Song, H. S. \& Zhang, S. Controlled generation of four-photon polarization-entangled decoherence-free states with conventional photon detectors. J. Opt. Soc. Am. B 26, 129 (2009).

55. Xia, Y., Song, J., Yang, Z. B. \& Zheng, S. B. Generation of four-photon polarization-entangled decoherence-free states within a network. Appl. Phys. B 99, 651 (2010).

56. Nemoto, K. \& Munro, W. J. Nearly deterministic linear optical controlled-NOT gate. Phys. Rev. Lett. 93, 250502 (2004).

57. Jeong, H. Using weak nonlinearity under decoherence for macroscopic entanglement generation and quantum computation. Phys. Rev. A 72, 034305 (2005).

58. Jeong, H. Quantum computation using weak nonlinearities: Robustness against decoherence. Phys. Rev. A 73, 052320 (2006).

59. Heo, J., Hong, C. H., Yang, H. J., Hong, J. P. \& Choi, S. G. Analysis of optical parity gates of generating Bell state for quantum information and secure quantum communication via weak cross-Kerr nonlinearity under decoherence effect. Quantum Inf. Process. 16, 10 (2017).

60. Lin, Q. \& Li, J. Quantum control gates with weak cross-Kerr nonlinearity. Phys. Rev. A 79, 022301 (2009).

61. He, B., Ren, Y. \& Bergou, J. A. Creation of high-quality long-distance entanglement with flexible resources. Phys. Rev. A 79, 052323 (2009).

62. He, B., Nadeem, M. \& Bergou, J. A. Scheme for generating coherent-state superpositions with realistic cross-Kerr nonlinearity. Phys. Rev. A 79, 035802 (2009).

63. Wittmann, C., Andersen, U. L., Takeoka, M., Sych, D. \& Leuchs, G. Discrimination of binary coherent states using a homodyne detector and a photon number resolving detector. Phys. Rev. A 81, 062338 (2010).

64. Lin, Q. \& He, B. Highly efficient processing of multi-photon states. Sci. Rep. 5, 12792 (2015).

65. Barrett, S. D. \& Milburn, G. J. Quantum-information processing via a lossy bus. Phys. Rev. A 74, 060302 (2006).

66. Lin, Q. \& He, B. Single-photon logic gates using minimal resources. Phys. Rev. A 80, 042310 (2009).

67. Munro, W. J., Nemoto, K., Beausoleil, R. G. \& Spiller, T. P. High-efficiency quantum-nondemolition single-photon-numberresolving detector. Phys. Rev. A 71, 033819 (2005).

68. Liu, Q., Wang, G. Y., Ai, Q., Zhang, M. \& Deng, F. G. Complete nondestructive analysis of two-photon six-qubit hyperentangled Bell states assisted by cross-Kerr nonlinearity. Sci. Rep. 6, 22016 (2016).

69. Costanzo, L. S. et al. Measurement-induced strong Kerr nonlinearity for weak quantum states of light. Phys. Rev. Lett. 119, 013601 (2017).

70. Hu, Y., Ge, G. Q., Chen, S., Yang, X. F. \& Chen, Y. L. Cross-Kerr-effect induced by coupled Josephson qubits in circuit quantum electrodynamics. Phys. Rev. A 84, 012329 (2011).

71. Zhang, H. \& Wang, H. Entanglement concentration of microwave photons based on the Kerr effect in circuit QED. Phys. Rev. A 95, 052314 (2017).

72. Kanamori, H. et al. Transmission characteristics and reliability of pure-silica-core single-mode fibers. J. Lightwave Technol. 4, 1144 (1986).

73. Nagayama, K. et al. Ultra low loss $(0.1484 \mathrm{~dB} / \mathrm{km})$ pure silica core fiber. SEI Tech. Rev. 57, 3 (2004).

74. Phoenix, S. J. D. Wave-packet evolution in the damped oscillator. Phys. Rev. A 41, 5132 (1990).

75. Sagona-Stophel, S., Shahrokhshahi, R., Jordaan, B., Namazi, M. \& Figueroa, E. Conditional $\pi$-phase shift of single-photon-level pulses at room temperature. Phys. Rev. Lett. 125, 243601 (2020).

76. Kok, P. Effects of self-phase-modulation on weak nonlinear optical quantum gates. Phys. Rev. A 77, 013808 (2008).

77. Lukin, M. D. \& Imamoğlu, A. Nonlinear optics and quantum entanglement of ultraslow single photons. Phys. Rev. Lett. 84, 1419 (2000).

78. Lukin, M. D. \& Imamoğlu, A. Controlling photons using electromagnetically induced transparency. Nature 413, 273 (2001).

79. Kok, P. et al. Linear optical quantum computing with photonic qubits. Rev. Mod. Phys. 79, 135 (2007).

\section{Acknowledgements}

This work was supported by the National Research Foundation of Korea(NRF) grant funded by the Korea government(MSIT) (No. 2021R1C1C2003302), by the National Research Foundation of Korea(NRF) grant funded by the Korea government(MSIT) (No. 2019R1A2C1006167), and by the MSIT(Ministry of Science and 
ICT), Korea, under the Grand Information Technology Research Center support program(IITP-2020-0-01462) supervised by the IITP(Institute for Information \& communications Technology Planning \& Evaluation).

\section{Author contributions}

J.H., conceived the main idea. J.H., wrote the manuscript and prepared all figures. J.H., calculated the main calculations. J.H., and S.G.C., analyzed the results. S.G.C., supervised the whole project. All authors reviewed the manuscript.

\section{Competing interests}

The authors declare no competing interests.

\section{Additional information}

Correspondence and requests for materials should be addressed to S.-G.C.

Reprints and permissions information is available at www.nature.com/reprints.

Publisher's note Springer Nature remains neutral with regard to jurisdictional claims in published maps and institutional affiliations.

(c) (i) Open Access This article is licensed under a Creative Commons Attribution 4.0 International License, which permits use, sharing, adaptation, distribution and reproduction in any medium or format, as long as you give appropriate credit to the original author(s) and the source, provide a link to the Creative Commons licence, and indicate if changes were made. The images or other third party material in this article are included in the article's Creative Commons licence, unless indicated otherwise in a credit line to the material. If material is not included in the article's Creative Commons licence and your intended use is not permitted by statutory regulation or exceeds the permitted use, you will need to obtain permission directly from the copyright holder. To view a copy of this licence, visit http://creativecommons.org/licenses/by/4.0/.

(C) The Author(s) 2021 